\title{
Post-buckling behaviour of prestressed steel stayed columns*
}

\author{
Daisuke Saito and M. Ahmer Wadee \\ Department of Civil and Environmental Engineering, \\ Imperial College of Science, Technology \& Medicine, \\ London SW7 2AZ, UK
}

\begin{abstract}
A steel column that is reinforced by prestressed stays generally has an increased strength in axial compression. A geometrically nonlinear model accounting for the post-buckling behaviour of the stayed column is formulated using the Rayleigh-Ritz method and then validated using the finite element method. It is found that the post-buckling behaviour is strongly linked to the level of the initial prestress. As the prestress is increased, the following different levels of the responses can be observed in sequence: initial Euler buckling that subsequently restabilizes strongly, the critical load increasing with a post-buckling path that is either stable or unstable, an upper limit for the critical load where the post-buckling is unstable after an initially rather flat response. These findings are important for designers aiming to achieve safer and more efficient designs for this structural component.
\end{abstract}

\section{Keywords}

Cable-supported structure; Nonlinear buckling; Rayleigh-Ritz method; Analytical modelling.

\section{Introduction}

A prestressed steel stayed column (Figure 1) is a structural component that is reinforced by either cable stays or rods such that its strength is increased in axial compression. Ordinary columns have a propensity to buckle under axial compression primarily due to their characteristic of being slender. To counter this, the prestressed steel stayed column is equipped with pre-tensioned stay systems; these restrain the column buckling displacement through

${ }^{*}$ Published in Engineering Structures: 30(5), pp. 1224-1239, 2008. 


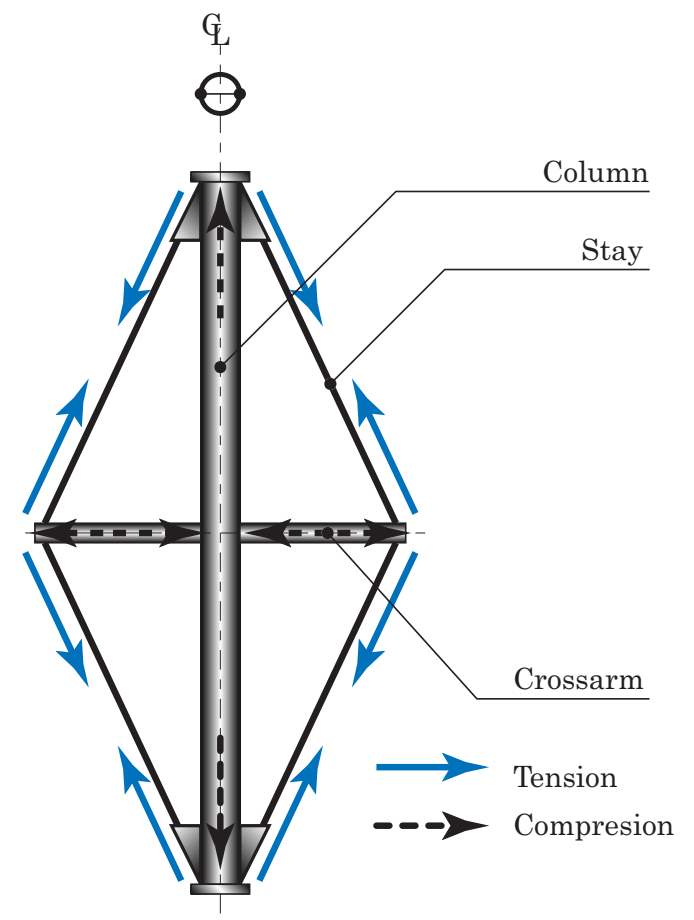

Figure 1: Principle of the prestress steel stayed column: stays are pretensioned to provide lateral restraint against overall buckling.

the horizontal crossarm placed at some intermediate distance from the column ends. Consequently, this additional system prevents the principal movement during conventional buckling and potentially provides a considerable increase in axial strength.

An application of this column type can be found where slender supports or towers are required, for example, it was used as a temporary support during the erection phase of the main stage of the "Rock in Rio III" stadium in Rio de Janeiro, Brazil [1, 2], (see Figure 2). In this project, it was required to support the large roof structures as high as 36 metres above ground level so that completion could be achieved within a limited time constraint. Conventionally, supporting the large roof structure at this height would have required a massive and complicated shoring system with a commensurate time penalty. To counter this the engineers decided to adopt the stayed column as the shoring system. Owing to its structural simplicity and superiority in resisting axial loads, this choice allowed the engineers to save significant time in the construction process.

In addition to these practical uses, a number of research works on the stayed column have existed since the 1960s, such as those evaluating critical buckling loads $[3,4,5,6,7,8]$, imperfection sensitivity studies [9, 10], and examining column's maximum axial strength $[11,12]$.

Despite this, as far as we are aware, the post-buckling response has not been investigated 


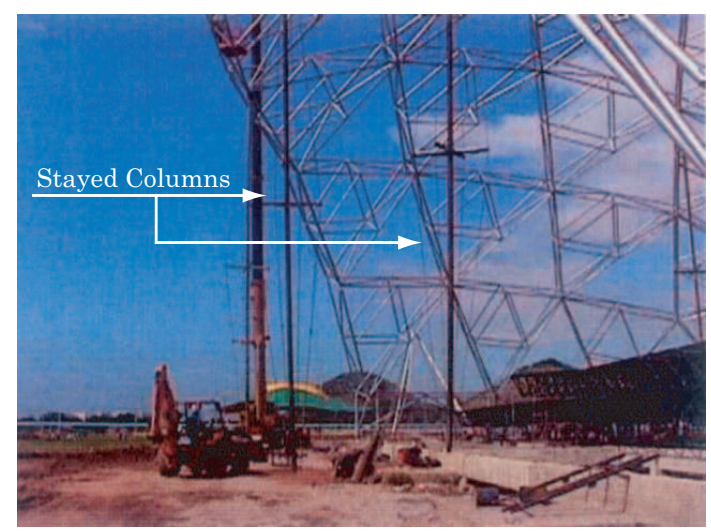

(a) stayed column as a temporary shoring

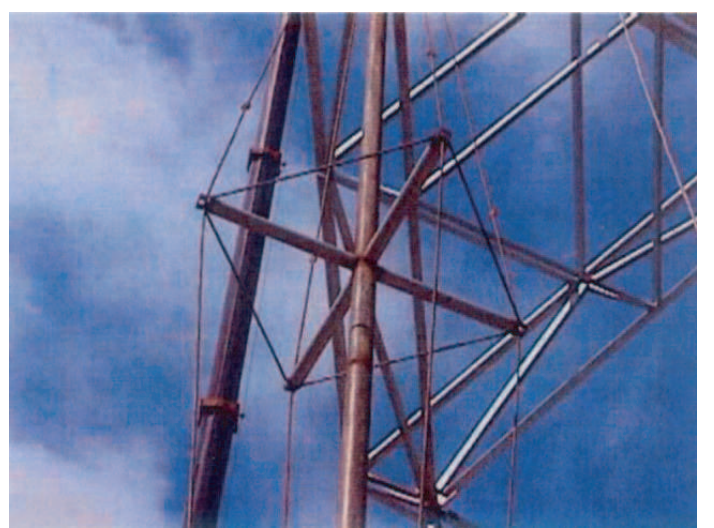

(b) crossarm in detail

Figure 2: Construction phase of Rock in Rio III main stage.

satisfactorily. This information is crucial to make the design safer and more efficient; stability in the post-buckling range implies that the design load could potentially be set higher than the critical buckling load; conversely instability in the post-buckling range means the design load should be reduced in order to ensure safety and the potentiality of the structure being sensitive to imperfections [13]. In the current study, the postbuckling response was investigated by developing analytical models using energy methods, the results of which were validated by the finite element method (FEM).

\subsection{Methodology}

In the current work, a single-crossarm stayed column, which was the simplest type, shown in Figure 3, was modelled. It is known from previous work that investigating the stayed column with an analytical procedure inevitably involves mathematically sophisticated formulations, therefore modelling the simplest structure is definitely a suitable first step to revealing its post-buckling response. Moreover, the majority of the literature deals with this single-crossarm type; hence validation and comparisons with previous research is possible.

In order to formulate the model, the total potential energy principle was applied in conjunction with the Rayleigh-Ritz method [13]. The total potential energy $V$ for the prestressed stayed column was developed as a multiple degree-of-freedom (MDOF) system. A set of algebraic equilibrium equations was derived from minimizing $V$ using the symbolic computation software MAPLE [14]. The structural response was revealed by this process, and was subsequently validated by the FEM using the well-established code ABAQUS [15]. For the analytical modelling, the following assumptions were made.

1. The column is simply-supported.

2. The connections between the stays and the column, and between the stays and the 


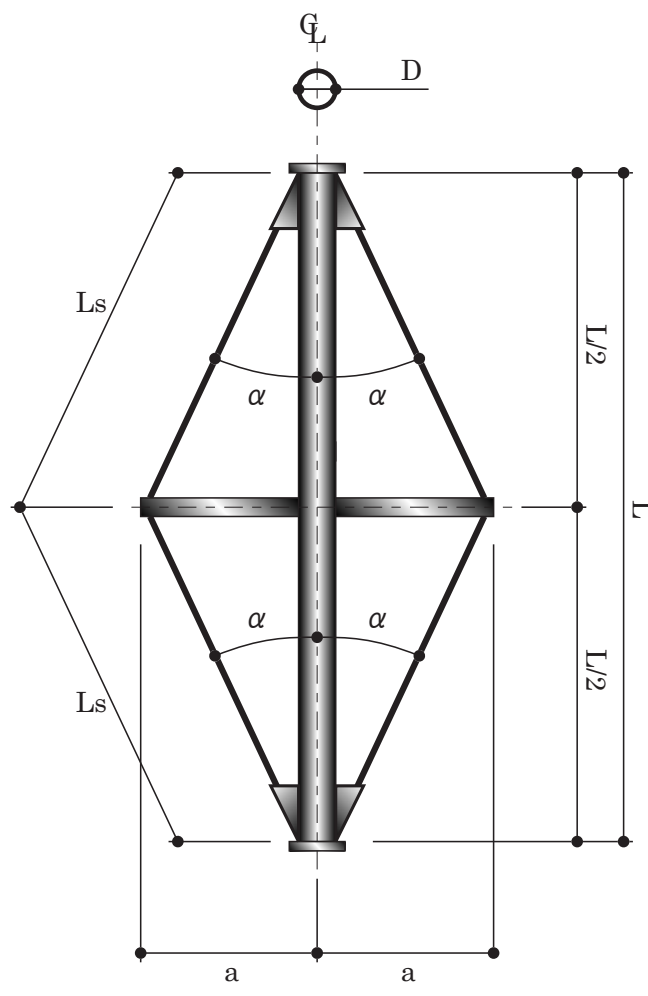

(a) Profile

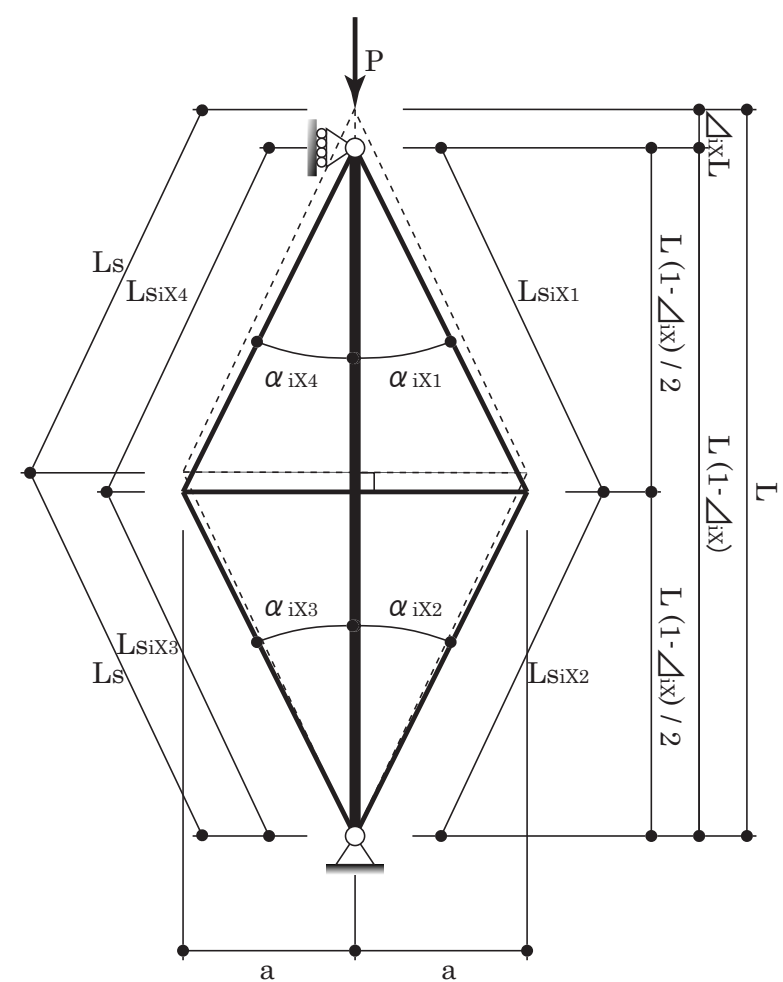

(b) Structural model

Figure 3: Structural model of the stayed column: column length $L$, crossarm length $a$, axial load $P$, the angle between the stay and the vertical $\alpha$ and the stay length $L_{s}$. The quantity $\Delta_{i X}$ represents the end-shortening of the column, where subscripts $i$ and $X$ represent a buckling mode number (1 or 2 ), and a buckling type $(A, B$ or $C$ ) respectively. Subscripts 1, 2, 3 and 4 after $X$ represent the number of the individual stays. Quantities $A, A_{s}$ and $A_{a}$ are defined as the cross sectional areas with $E, E_{s}$ and $E_{a}$ being the Young's moduli of the column, the stays and the crossarm respectively. The quantities $I$ and $I_{a}$ refer to the cross-sectional second moment of area of the column and the crossarm respectively 
crossarms, are ideal hinges. The connections between the crossarm and the column are rigid.

3. The column is centrally loaded and perfectly straight, i.e. imperfections are not taken into account in the analysis.

4. The axial deformation of the crossarm and the bending deformation of the stays are both ignored.

5. The stay goes slack the instant it goes into compression; hence it does not carry any stresses in compression.

6. The analysis is purely elastic; hence, the stress-strain relationship is completely linear apart from the stay slackening.

7. Changes in geometries from applying the prestress are ignored, i.e. the initial configuration is kept after the introduction of the initial prestress.

Changes in geometries from the prestress do not yield significant effects unless the initial prestress has the same level as the Euler load of the column. As this level of prestress leads to a considerable amount of compressive force in the column, which significantly diminishes the axial buckling resistance, this situation is considered to be impractical.

\section{Model Formulation}

In this section, the MDOF system is developed by considering, in turn, the displacements of each component and the geometrical changes after applying the prestress. This leads to the total potential energy function.

\subsection{Displacement functions for the column}

Two different buckling mode shapes for the column are considered: a symmetric shape (Mode 1) and an antisymmetric shape (Mode 2) about the column mid-span, as shown in Figure 4; these are the basic possible deflection shapes for buckling in the single-crossarm stayed column. In Mode 1, the maximum curvature can be found at the column midspan and zero curvature at both ends; in Mode 2, zero curvature can be found at the column midspan and both ends. Each mode can be expressed as a summation of sinusoidal waves. Defining the column length as $L$ (see Figure 3) and the generalized coordinates as $q_{m}$, where the subscript $m$ is an integer representing a degree of freedom for a sinusoidal wave that has a wavelength of $2 L / m$, the displacement functions for the column $W_{1}$ and $W_{2}$ can 
Mode 1

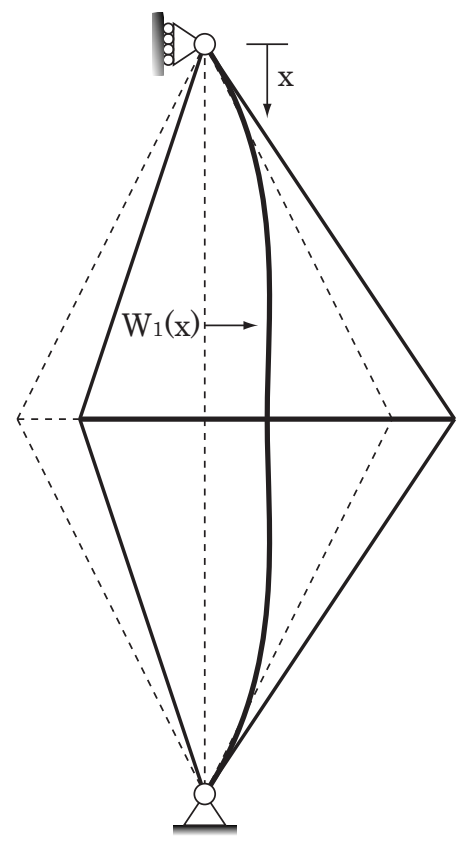

Mode 2

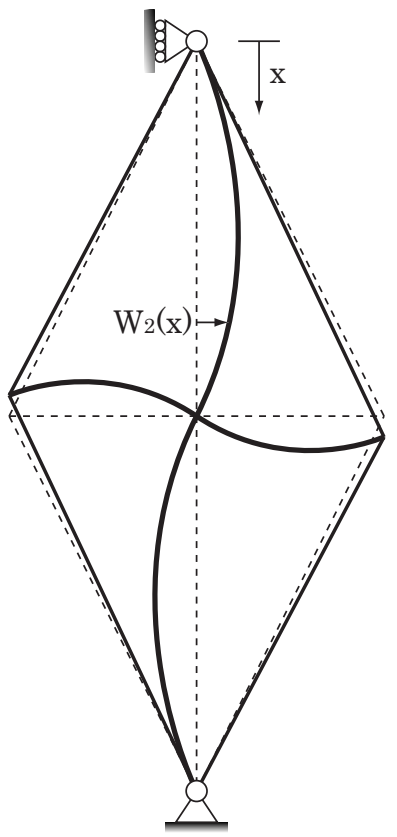

Figure 4: Buckling Modes 1 (symmetric) and 2 (antisymmetric).

be assumed to be as follows:

$$
\begin{aligned}
& W_{1}(x)=q_{1} L \sin \frac{\pi x}{L}+q_{3} L \sin \frac{3 \pi x}{L}+\cdots=\sum_{m=1}^{n} q_{2 m-1} L \sin \frac{(2 m-1) \pi x}{L}, \\
& W_{2}(x)=q_{2} L \sin \frac{2 \pi x}{L}+q_{4} L \sin \frac{4 \pi x}{L}+\cdots=\sum_{m=1}^{n} q_{2 m} L \sin \frac{2 m \pi x}{L}
\end{aligned}
$$

where $n$ represents the number of degrees of freedom in the model. As the individual components of the stayed column tend to be long and thin, Euler-Bernoulli bending theory can be applied; the angles of the members to the vertical $\Theta_{1}(x)$ and $\Theta_{2}(x)$ are therefore approximated as the first derivative of the displacement with respect to $x$ :

$$
\begin{gathered}
\Theta_{1}(x)=q_{1} \pi \cos \frac{\pi x}{L}+3 \pi q_{3} \cos \frac{3 \pi x}{L}+\cdots=\sum_{m=1}^{n}(2 m-1) q_{2 m-1} \pi \cos \frac{(2 m-1) \pi x}{L} \\
\Theta_{2}(x)=2 q_{2} \pi \cos \frac{2 \pi x}{L}+4 \pi q_{4} \cos \frac{4 \pi x}{L}+\cdots=\sum_{m=1}^{n} 2 m q_{2 m} \pi \cos \frac{2 m \pi x}{L}
\end{gathered}
$$




\subsection{Displacement functions for the crossarm}

\subsubsection{Buckling type distinction}

The deflected shape of the crossarm and the function for the end shortening of the column depend on the stress state of the stays (see Figure 5). To take these effects into account

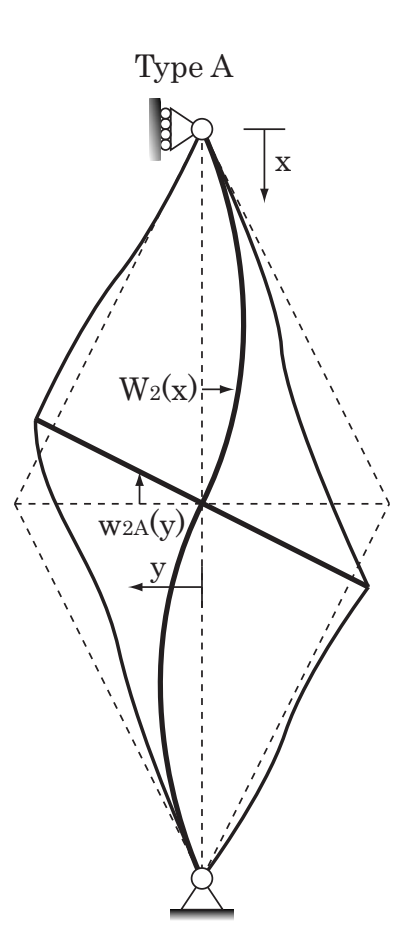

Mode 2

Type B

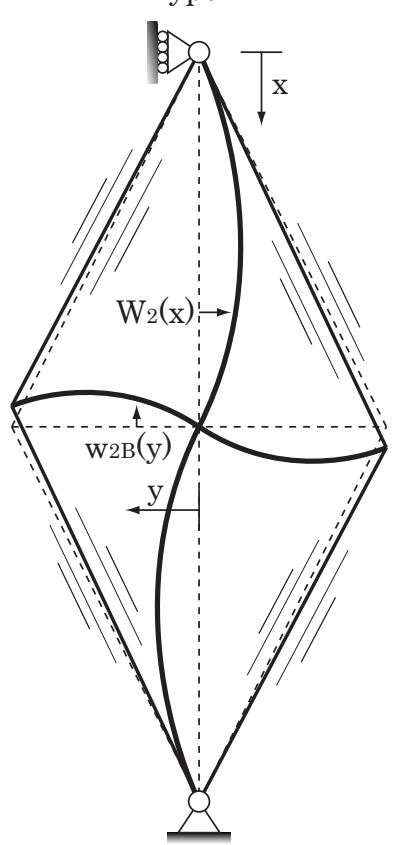

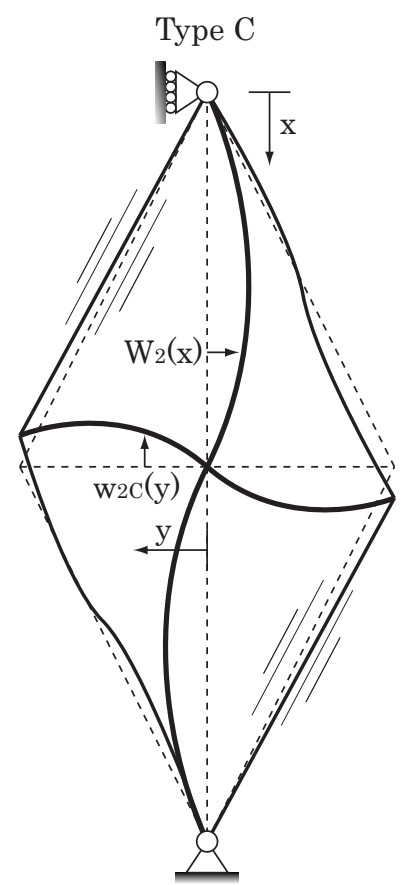

Figure 5: Buckling types in Mode 2.

in the current model, the following four states are considered:

1. Type A: all of the stays are slack.

2. Type B: all of the stays are active.

3. Type C: two stays are active.

Note that Type A occurs with a small value of the initial prestress; Type B occurs with a sufficient amount of the initial prestress which allows the stays not to slacken until buckling; Type C buckling can occur either after Type A, B or the fundamental (pre-buckling) state. Shape functions for the crossarm for each type can be obtained by solving the differential equations reflecting each type of stress state in the stays and the reaction forces developed in the crossarm. 


\subsubsection{Shape functions}

First, the bending moment for $y \geqslant 0$ in the crossarm (see Figure 6) $M_{a B}$ is given by

$$
M_{a X}=-R_{h X}\left[h_{X}-w_{2 X}(y)\right]+R_{v X}(a-y),
$$

where $R_{h X}$ and $R_{v X}$ are horizontal and vertical reaction forces respectively at the tip of the crossarm; $h_{X}$ is the displacement at the tip of the crossarm; $y$ is the horizontal axis; and $w_{2 X}(y)$ is the deflection of the crossarm perpendicular to the coordinate. Ignoring higher-

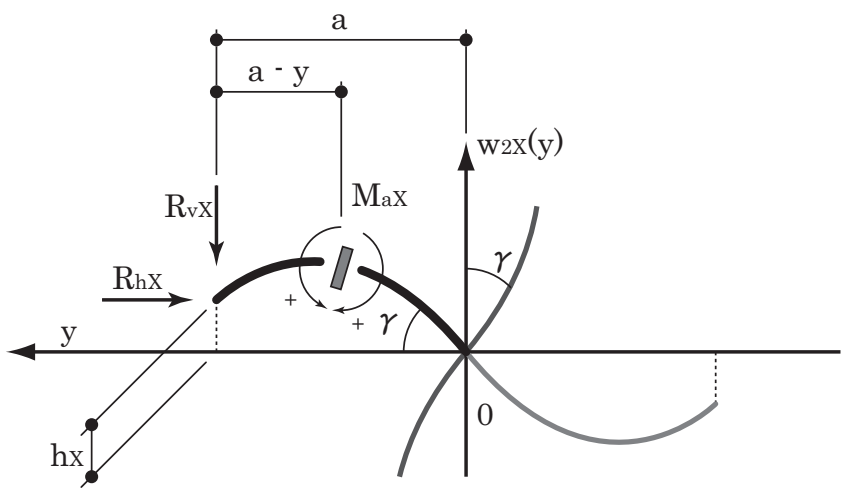

Figure 6: Free body diagram to determine the bending moment at an arbitrary cross section $(y \leqslant 0)$ of the crossarm. Note that the subscript $X$ represents the buckling type classification which can be either $B$ or $C$.

order terms and the effect of the end-shortening of the crossarm, the basic differential equation for the bending of the crossarm takes the form:

$$
M_{a X}=-E_{a} I_{a} w_{2 X}^{\prime \prime}(y),
$$

where primes represent differentiation with respect to $y$, and $E_{a}$ and $I_{a}$ are the Young's modulus and the cross-sectional second moment of area of the crossarm respectively. Substituting equation (6) into (5) leads to

$$
w_{2 X}^{\prime \prime}(y)+k_{X}^{2} w_{2 X}(y)=-\frac{R_{v X}}{E_{a} I_{a}}(a-y)+h k_{X}^{2},
$$

where

$$
k_{X}=\sqrt{\frac{R_{h X}}{E_{a} I_{a}}} .
$$

The general solution of equation (7) is

$$
w_{2 X}(y)=H_{X} \sin k_{X} y+K_{X} \cos k_{X} y-\frac{R_{v X}}{k_{X}^{2} E_{a} I_{a}}(a-y)+h_{X},
$$


where $H_{X}$ and $K_{X}$ are constants of integration that are determined from the boundary conditions, thus:

$$
w_{2 X}(0)=0, \quad w_{2 X}^{\prime}(0)=\gamma, \quad w_{2 X}(a)=h_{X},
$$

where $\gamma$ is the angle between the horizontal and the crossarm at the mid-point, defined as

$$
\gamma=-\Theta_{2}(L / 2)=2 q_{2} \pi-4 q_{4} \pi+\cdots=\sum_{m=1}^{n}(-1)^{m-1} 2 m q_{2 m} \pi .
$$

The second condition comes from the assumption that $W_{2}(x)$ intersects the crossarm at right angles. Applying this condition yields the following expressions:

$$
\begin{aligned}
H_{X} & =\frac{1}{k_{X}}\left(-\frac{R_{v X}}{k_{X}^{2} E_{a} I_{a}}+\gamma\right), \quad K_{X}=\frac{R_{v X} a}{k_{X}^{2} E_{a} I_{a}}-h_{X}, \\
h_{X} & =\frac{\left(\gamma E_{a} I_{a} k_{X}^{2}-R_{v X}\right) \sin k_{X} a+k_{X} R_{v X} a \cos k_{X} a}{k_{X}^{3} E_{a} I_{a} \cos k_{X} a} .
\end{aligned}
$$

In order to find the actual shape of the crossarm with equation (9), it is also necessary to establish equations for $R_{v X}$ and $R_{h X}$. With reference to Figure 7 and then by taking the leading terms of $\Delta_{2 X}$ and $h$, the changes in the axial force in Stay 3 and Stay $4, \delta F_{X 3}$ and

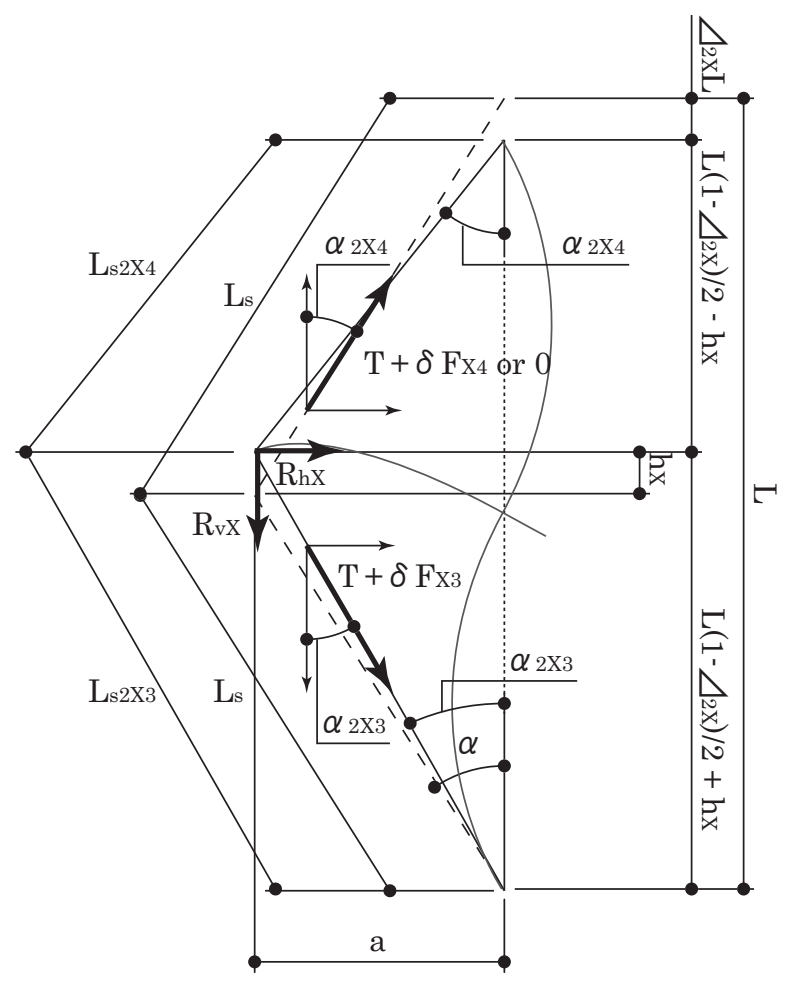

Figure 7: Elongation of the stays and reaction forces at the tip of the crossarm. 
$\delta F_{X 4}$ respectively, resulting from the structural displacement can be expressed as follows:

$$
\begin{aligned}
& \delta F_{X 3}=E_{s} A_{s} \frac{L_{s 2 X 3}-L_{s}}{L_{s}} \approx\left(-\Delta_{2 X}+\frac{2 h}{L}\right) \cos ^{2} \alpha, \\
& \delta F_{X 4}=E_{s} A_{s} \frac{L_{s 2 X 4}-L_{s}}{L_{s}} \approx-\left(\Delta_{2 X}+\frac{2 h}{L}\right) \cos ^{2} \alpha .
\end{aligned}
$$

From the expression for $\delta F_{X 3}$ and $\delta F_{X 4}$, the vertical and the horizontal reaction forces for Type B, $R_{v B}$ and $R_{h B}$, can be obtained thus:

$$
\begin{aligned}
& R_{v B}=\left(T+\delta F_{B 3}\right) \cos \alpha_{2 B 3}-\left(T+\delta F_{B 4}\right) \cos \alpha_{2 B 4}=\frac{4 h}{L}\left(T \sin ^{2} \alpha+E_{s} A_{s} \cos ^{2} \alpha\right) \cos \alpha, \\
& R_{h B}=\left(T+\delta F_{B 3}\right) \sin \alpha_{2 B 3}+\left(T+\delta F_{B 4}\right) \sin \alpha_{2 B 4}=2\left[T+\left(T-E_{s} A_{s}\right) \Delta_{2 B} \cos ^{2} \alpha\right] \sin \alpha .
\end{aligned}
$$

As only one stay is active on each side in Type C, $R_{v C}$ and $R_{h C}$, can thus be obtained from $\delta F_{X 3}$. However, including the $h_{C}$ term in the $R_{h C}$ equation causes a computation problem that leaves the governing equation untractable. To rectify this we apply the approximation $h=0$ in $R_{h C}$, which applies when the stays first slacken, thereby enabling us to obtain $R_{h C}$ :

$$
\begin{aligned}
R_{v C} & =\left(T+\delta F_{C 3}\right) \cos \alpha_{2 C 3}=\left[1-\left(\Delta_{2 C}-\frac{2 h_{C}}{L}\right)\left(\sin ^{2} \alpha+\frac{E_{s} A_{s}}{T} \cos ^{2} \alpha\right)\right] T \cos \alpha, \\
R_{h C} & =\left(T+\delta F_{C 3}\right) \sin \alpha_{2 C 3}=\left[1+\left(\Delta_{2 C}-\frac{2 h_{C}}{L}\right)\left(1-\frac{E_{s} A_{s}}{T}\right) \cos ^{2} \alpha\right] T \sin \alpha \\
& \approx\left[\left(1+\Delta_{2 C} \cos ^{2} \alpha\right) T-E_{s} A_{s} \Delta_{2 C} \cos ^{2} \alpha\right] \sin \alpha .
\end{aligned}
$$

\subsection{Stress and geometrical changes in the structure}

Stress and geometrical changes in the structure are investigated prior to the energy formulation presented in the following section. The investigation includes such items as the stress changes by the prestress, the elongation of the stays and the end-shortening of the column.

\subsubsection{Initial stress of the column with prestress}

With reference to Figure 8, the initial prestresses that are introduced to the column $T_{c}$ and the crossarm $T_{a}$ are

$$
T_{c}=2 T \cos \alpha, \quad T_{a}=2 T \sin \alpha .
$$

Therefore, the strains in the stay $\varepsilon_{s t}$, the column $\varepsilon_{c t}$, and the crossarm $\varepsilon_{a t}$ are respectively:

$$
\varepsilon_{s t}=\frac{T}{E_{s} A_{s}}, \quad \varepsilon_{c t}=\frac{2 T \cos \alpha}{E A}, \quad \varepsilon_{a t}=\frac{2 T \sin \alpha}{E_{a} A_{a}} .
$$




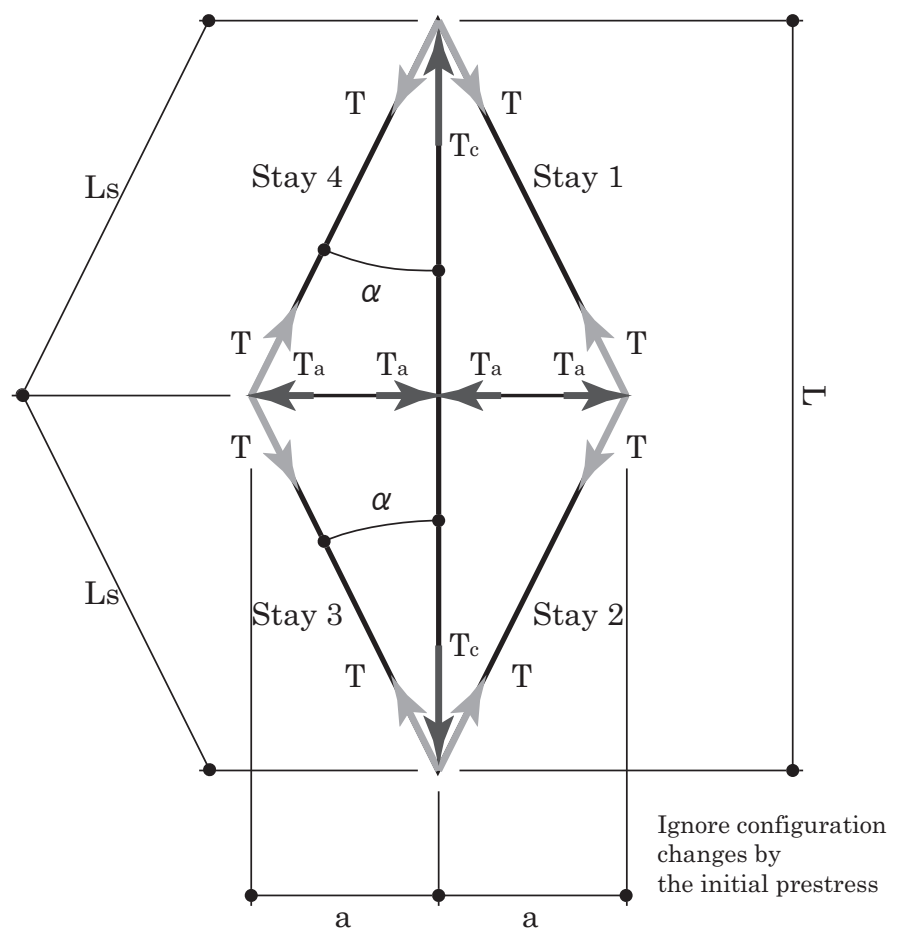

Figure 8: Effect of the initial prestress.

\subsubsection{Tip displacement coefficient}

The tip displacement of the crossarm is necessary to find the elongation of the stays. The tip displacement for Type B can also be obtained from $h_{B}$, which is given in equation (10). Expanding $h_{B}$ the fifth order with respect to $R_{B}$ and then taking the leading order with respect to $\Delta_{2 B}$ and $\gamma$ yield

$$
h_{B}=c_{B} a \gamma,
$$

where $c_{B}$ is the factor expressing the magnitude of the tip displacement of the crossarm for Type B:

$$
c_{B}=\left[1+\frac{2 E_{s} A_{s}}{3 E_{a} I_{a}} a^{2} \sin \alpha \cos ^{2} \alpha\right]^{-1} .
$$

and $R_{B}$ is

$$
R_{B}=a \sqrt{\frac{2\left[\left(1+\Delta_{2 B} \cos ^{2} \alpha\right) T-E_{s} A_{s} \Delta_{2 B} \cos ^{2} \alpha\right] \sin \alpha}{E_{a} I_{a}}} .
$$

The same value of tip displacement can be obtained using the work of Smith et al. [5]. The tip displacement for Type $\mathrm{C}$ can also be obtained from $h_{C}$, which is given in equation (10). However, as the direct expression that can be obtained from $h_{C}$ is too complicated for the analytical model, this is simplified by using the Taylor expansion to the fifth order 
with respect to $R_{C}$. Then taking leading order with respect to $\Delta_{2 C}$ and $\gamma$ such that $h_{C}$ is

$$
h_{C}=c_{C} a \gamma+c_{C \Delta} a \Delta_{2 C}+c_{C 0} a,
$$

where $c_{C}, c_{C \Delta}$ and $c_{C 0}$ are the factor expressing the magnitude of the tip displacement of the crossarm in Type C:

$$
\begin{gathered}
c_{C}=\frac{\left(15 E_{a} I_{a}-E_{s} A_{s} a^{2} \sin \alpha\right) a^{2} T \sin \alpha \cos ^{2} \alpha+15 \zeta E_{a} I_{a}}{5 \zeta^{2}} \\
c_{C \Delta}=\frac{\left[\left(3 E_{a} I_{a}+2 E_{s} A_{s} a^{2} \sin \alpha \cos ^{2} \alpha\right) T \sin ^{2} \alpha+\zeta E_{s} A_{s} \cos ^{2} \alpha\right] a^{2} \cos \alpha}{\zeta^{2}} \\
c_{C 0}=-\frac{a^{2} T \cos \alpha}{\zeta},
\end{gathered}
$$

where

$$
\zeta=\left(3 E_{a} I_{a}+E_{s} A_{s} a^{2} \sin \alpha \cos ^{2} \alpha\right)
$$

and $R$ is

$$
R_{C}=a \sqrt{\frac{\left[1+\left(1-\frac{E_{s} A_{s}}{T}\right) \Delta_{2 C} \cos ^{2} \alpha\right] T \sin \alpha}{E_{a} I_{a}}} .
$$

Note that this simplification becomes less accurate when the initial prestress $T$ is large.

\subsubsection{Elongation of the stays}

The post-buckling shapes are sketched in Figure 9; these geometries allow the new stay length $L_{s i X j}$, where the subscript $j$ refers to the stay number as indicated in Figure 8, to be evaluated through Pythagoras's theorem, which leads to the strain in the stays purely arising from the applied load $P$ in the stays. Subsequently, this equation is expanded as a Taylor series up to second order with respect to $q_{m}$ and $\Delta_{i X}$. In this process, the cross and quadratic terms of $\Delta_{i X}$ such as $\Delta_{i X} q_{m}$ and $\Delta_{i X}^{2}$ are dropped, as these terms are considered to be small. By combining the expanded strain $\varphi_{i X j}$ with the initial prestress $T$, the total strains in the stays $\varepsilon_{s i X j}$ can be obtained, giving the following expressions:

$$
\varepsilon_{s i X j}=\varphi_{i X j}+\varepsilon_{s t} .
$$

\subsubsection{End-shortening of the column}

In order to find the end-shortening expression of the column $\Delta_{i X}$, equilibrium is considered at the end of the column where the external load $P$ is applied with the free body diagram approach shown in Figure 10. Vertical force equilibrium and moment equilibrium around the point $O$ give the following equations:

$$
\begin{array}{r}
T_{i X 1} \cos \alpha_{i X 1}+T_{i X 4} \cos \alpha_{i X 4}+P-C_{i X} \cos \beta_{i}-S_{i X} \cos \beta_{i}=0, \\
M_{i X}-\mathrm{d} x S_{i X} \cos \beta_{i X}-W_{i}(\mathrm{~d} x) S_{i X} \sin \beta_{i}-W_{i}(\mathrm{~d} x) C_{i X} \cos \beta_{i}+\mathrm{d} x C_{i X} \sin \beta_{i}=0,
\end{array}
$$




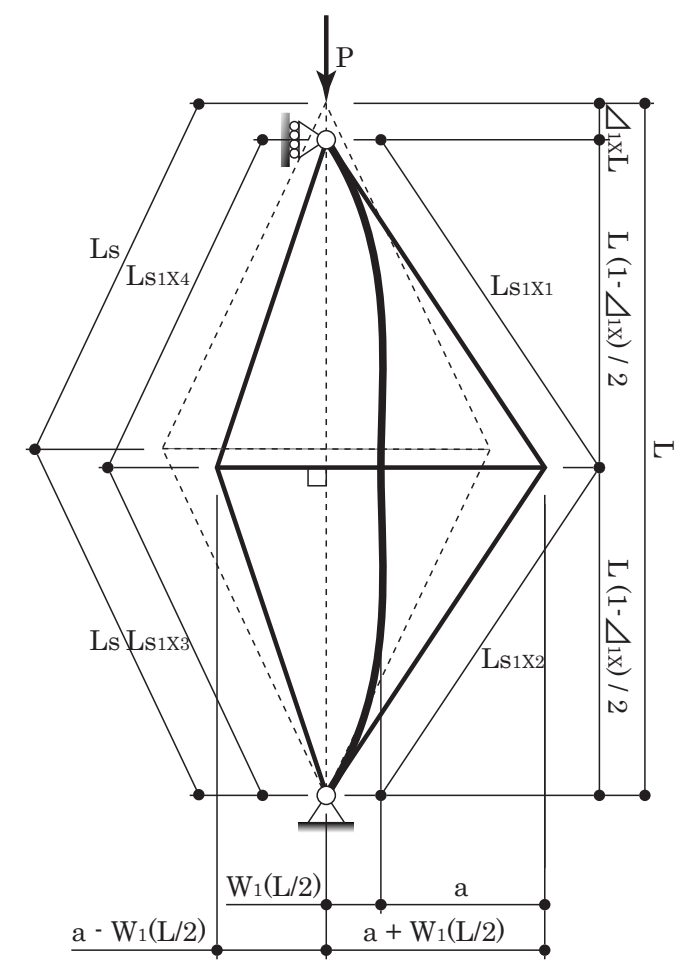

(a) Mode 1

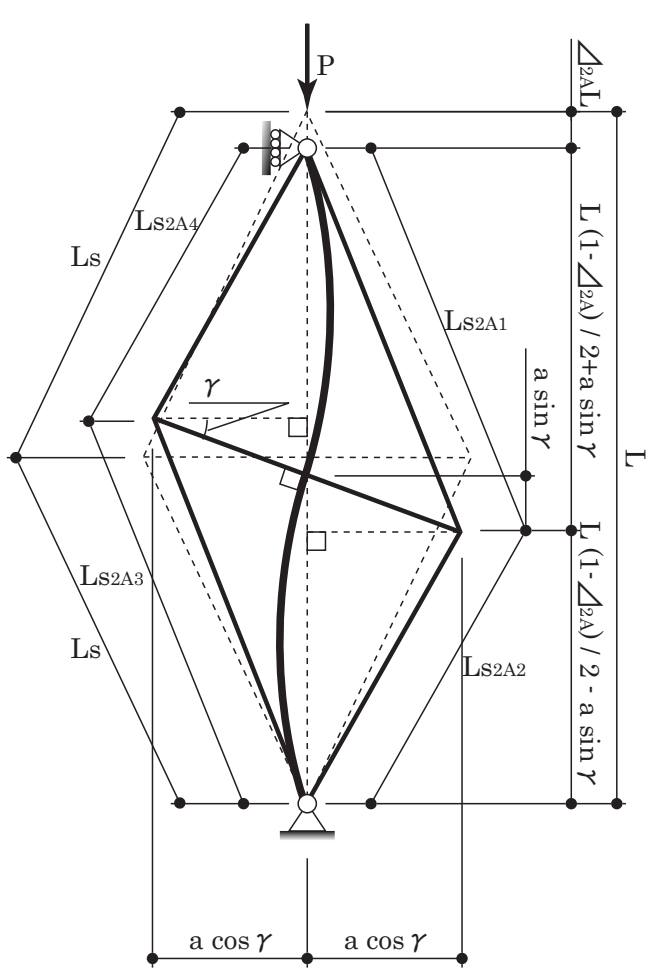

(b) Mode 2 Type A

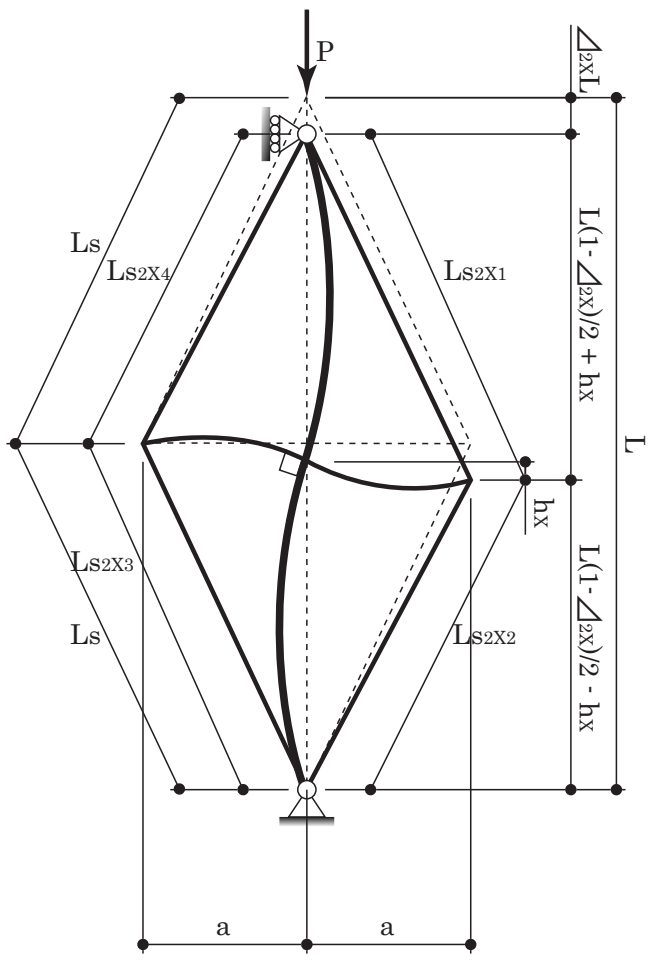

(c) Mode 2 Type B-C

Figure 9: Geometry of the stayed column in buckling modes 1 and 2. 


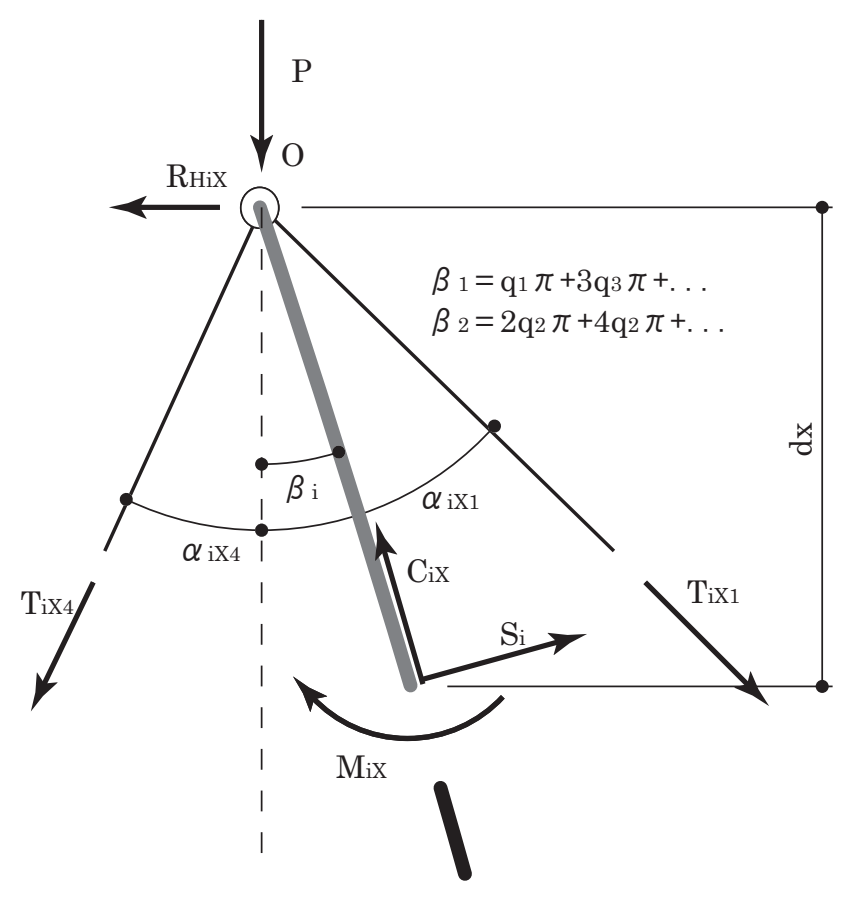

Figure 10: Equilibrium free body diagram for the column. Note that $R_{H i X}$ is the horizontal reaction force at the end of the column.

where $T_{i X j}$ is the axial force in stay $j$; with $C_{i X}, S_{i X}$ and $M_{i}$ being an axial force, a shear force and a bending moment respectively in the column at a point which is a small distance $\mathrm{d} x$ away from $O ; \beta_{i}$ is an angle between the column and the vertical; $\alpha_{i X 1}$ and $\alpha_{i X 4}$ are angles between each stay and the vertical. These angles, internal forces and moments need to be defined in order to solve the equilibrium equations and to obtain an expression for $\Delta_{i X}$. Firstly, $\beta_{i}$ can be obtained by substituting $x=0$ into $\Theta_{i}(x)$ defined in equations (3) and (4):

$$
\begin{aligned}
& \beta_{1}=\Theta_{1}(0)=q_{1} \pi+3 q_{1} \pi+\cdots=\sum_{m=1}^{n}(2 m-1) q_{2 m-1} \pi \\
& \beta_{2}=\Theta_{2}(0)=2 q_{2} \pi+4 q_{1} \pi+\cdots=\sum_{m=1}^{n} 2 m q_{2 m} \pi .
\end{aligned}
$$

With reference to Figures $9(\mathrm{a})-(\mathrm{c}), \cos \alpha_{i X 1}$ and $\cos \alpha_{i X 4}$ are obtained through trigonometry; subsequently, those relationships are expressed to the leading order with respect to 
$q_{m}$ and $\Delta_{i X}$. For example, $\cos \alpha_{1 X 1}$ is given as

$$
\begin{aligned}
\cos \alpha_{1 X 1} & =\frac{\frac{1}{2} L\left(1-\Delta_{1 X}\right)}{\sqrt{\left[\frac{1}{2} L\left(1-\Delta_{1 X}\right)\right]^{2}+\left[\sum_{m=1}^{n} L(-1)^{m-1} q_{2 m-1}+a\right]^{2}}} \\
& \approx\left(1-\Delta_{1 X} \sin ^{2} \alpha\right) \cos \alpha .
\end{aligned}
$$

As all of the required angles are defined, the forces and moments $T_{i X}, C_{i X}, S_{i X}$, and $M_{i X}$ in the free body diagram need to be investigated. Firstly, with the strain expressions of the stays shown in the previous section and the assumption that the stays do not resist compression, the axial forces in the stays $T_{i X 1}$ and $T_{i X 4}$ are defined as follows:

$$
\begin{aligned}
& T_{i A 1}=T_{i A 4}=T_{i C 4}=0, \\
& T_{i B 1}=\varepsilon_{i B 1} E_{s} A_{s}, \quad T_{i B 4}=\varepsilon_{i B 4} E_{s} A_{s}, \quad T_{i C 1}=\varepsilon_{i C 1} E_{s} A_{s} .
\end{aligned}
$$

The axial strain in the column $\varepsilon_{c 1 X}$ is expressed as a summation of the components $\Delta_{i X}$ and $\varepsilon_{c t}$ minus the effect of the relaxation from the buckling displacement. Therefore, the axial strain for each mode is expressed as follows:

$$
\begin{aligned}
& \varepsilon_{c 1 X}=\Delta_{1 X}+\varepsilon_{c t}-\frac{1}{L} \int_{0}^{L} \frac{1}{2} W_{1}^{\prime 2}(x) \mathrm{d} x=\Delta_{1 X}+\frac{2 T \cos \alpha}{E A}-\sum_{m=1}^{n} \frac{(2 m-1)^{2} \pi^{2} q_{2 m-1}^{2}}{4}, \\
& \varepsilon_{c 2 X}=\Delta_{2 X}+\varepsilon_{c t}-\frac{1}{L} \int_{0}^{L} \frac{1}{2} W_{2}^{\prime 2}(x) \mathrm{d} x=\Delta_{2 X}+\frac{2 T \cos \alpha}{E A}-\sum_{m=1}^{n} m^{2} \pi^{2} q_{2 m}^{2}
\end{aligned}
$$

Thus, the axial force $C_{i X}$ is described as

$$
C_{i X}=E A \varepsilon_{c i X}
$$

With linear bending theory, the bending moments $M_{i}$ are expressed as the following equations:

$$
\begin{aligned}
& M_{1}=-E I W_{1}^{\prime \prime}(\mathrm{d} x)=\sum_{m=1}^{n} \frac{(2 m-1)^{2} \pi^{2} E I q_{2 m-1}}{L} \sin \frac{(2 m-1) \pi \mathrm{d} x}{L} \\
& M_{2}=-E I W_{2}^{\prime \prime}(\mathrm{d} x)=\sum_{m=1}^{n} \frac{(2 m)^{2} \pi^{2} E I q_{2 m}}{L} \sin \frac{2 m \pi \mathrm{d} x}{L} .
\end{aligned}
$$

The shear force $S_{i X}$ can be defined by substituting equations (39) and either equation (40) for Mode 1 or equation (41) for Mode 2 into equation (32) and then by taking the limit $\mathrm{d} x \rightarrow 0$.

By substituting equations (36), (39) and an expression for the shear force, either (40) for Mode 1 or (41) for Mode 2, into equation (31), the expression for $\Delta_{i X}$ can be obtained. 
Subsequently, the solution is expressed as a Taylor series with respect to $T, P$ and $q_{m}$ up to second order, which gives the following simplified equations:

$$
\begin{aligned}
\Delta_{1 X} & =b_{p X} P+b_{t X} T+b_{1 X} q_{1}+b_{3 X} q_{3}+\cdots+b_{11 X} q_{1}^{2}+b_{13 X} q_{1} q_{3}+b_{33 X}^{2} q_{3}^{2}+\cdots \\
& =b_{p X} P+b_{t X} T+\sum_{m=1}^{n} b_{2 m-1 X} q_{2 m-1}+\sum_{m=1, l=1, m \leqslant l}^{n} b_{2 m-12 l-1 X} q_{2 m-12 l-1} q_{2 m-12 l-1}, \\
\Delta_{2 X} & =b_{p X} P+b_{t X} T+b_{2 X} q_{2}+b_{4 X} q_{4}+\cdots+b_{22 X} q_{2}^{2}+b_{24 X} q_{2} q_{4}+b_{44 X}^{2} q_{4}^{2}+\cdots \\
& =b_{p X} P+b_{t X} T+\sum_{m=1}^{n} b_{2 m X} q_{2 m}+\sum_{m=1, l=1, m \leqslant l}^{n} b_{2 m 2 l X} q_{2 m 2 l} q_{2 m 2 l} .
\end{aligned}
$$

where $b_{p X}, b_{t X}, b_{m X}$ and $b_{m l X}$ are coefficients for $P, T, q_{m}$, and $q_{m} q_{l}$ respectively.

\subsection{Energy formulation}

The total potential energy $V_{i X}$ comprises components of strain energy and the work done by the load. In a general state of deflection, there are four components of strain energy: from bending in the column $\left(U_{c b i}\right)$ and the crossarm $\left(U_{a b i X}\right)$ with axial strains in the column $\left(U_{\text {caiX }}\right)$ and stays $\left(U_{s i X}\right)$. Note that the bending energy in the crossarm $\left(U_{a b i X}\right)$ only exists in Mode 2 in buckling Types $\mathrm{B}$ and $\mathrm{C}$ as the crossarm does not bend in the other cases.

\subsubsection{Bending energy}

The bending energy components in the column arise from a linear curvature expression; thus, $W_{i}$ give following expressions for $U_{c b i}$ :

$$
\begin{aligned}
& U_{c b 1}=\frac{1}{2} E I \int_{0}^{L} W_{1}^{\prime \prime 2}(x) \mathrm{d} x-U_{c b 0}=\sum_{m=1}^{n} \frac{(2 m-1)^{4} E I q_{2 m-1}^{2} \pi^{4}}{4 L}-U_{c b 0}, \\
& U_{c b 2}=\frac{1}{2} E I \int_{0}^{L} W_{2}^{\prime \prime 2}(x) \mathrm{d} x-U_{c b 0}=\sum_{m=1}^{n} \frac{(2 m)^{4} E I q_{2 m}^{2} \pi^{4}}{4 L}-U_{c b 0},
\end{aligned}
$$

where $U_{c b 0}$ is the existing column bending energy at the beginning of each buckling type.

In a similar way, the bending energy in the crossarm for Mode 2 Types B and $\mathrm{C}$ can be obtained. Note that crossarm symmetry accounts for the doubling of the standard bending energy expression:

$$
\begin{aligned}
U_{a b 2 X}= & E_{a} I_{a} \int_{0}^{a} w_{2 X}^{\prime \prime 2}(y) \mathrm{d} y-U_{a b 0} \\
= & E_{a} I_{a} k_{B}^{3}\left\{2 H_{X} K_{X}-H_{X}^{2} \cos k_{X} a \sin k_{X} a+H_{X}^{2} k_{X} a-2 H_{X} K_{X} \cos ^{2} k_{X} a\right. \\
& \left.+K_{X}^{2} \cos k_{X} a \sin k_{X} a+K_{X}^{2} k_{X} a\right\} / 2-U_{a b 0},
\end{aligned}
$$

where $U_{a b 0}$ is the existing crossarm bending energy at the beginning of each buckling type. Note that $U_{c b 0}$ and $U_{a b 0}$ have independent values from $q_{m}$, therefore they do not affect the critical load nor the post-buckling path as it simply vanishes on differentiation. 


\subsubsection{Axial energy}

The axial energy $U_{\text {caiX }}$ in the column accounts for the energy gained through the axial compression from the load $P$ together with the effect of the relaxation from the buckling displacement; using equations (37) and (38) as the ending points of integration for each mode, the axial energy is obtained as

$$
U_{c a i X}=\int_{\varepsilon_{c X 0}}^{\varepsilon_{c i X}} E A L \varepsilon \mathrm{d} \varepsilon=\frac{1}{2} E A L\left(\varepsilon_{c i X}^{2}-\varepsilon_{c X 0}^{2}\right),
$$

where $\varepsilon_{c X 0}$ is the existing strain at the beginning of each type.

The axial energy in the stays is obtained by integrating the stress-strain relationship over the stay volume - written as the product of the cross-sectional area $A_{s}$ and the length $L_{s}$ :

$$
U_{s i X}=\sum_{j=1}^{4} U_{s i X j}=\sum_{j=1}^{4} \int_{\varepsilon_{s X 0}}^{\varepsilon_{s i X j}} A_{s} L_{s} \sigma\left(\varepsilon_{s i X j}\right) \mathrm{d} \varepsilon,
$$

where $U_{s i X j}$ is the strain energy stored in stay $j$ for Mode $i$ Type $X$; $\varepsilon_{s X 0}$ is the existing strain at the commencement of each type. The stress-strain curve of the stays is assumed to be piecewise linear thus:

$$
\sigma_{s}\left(\varepsilon_{s i X j}\right)=\left\{\begin{array}{lll}
E_{s} \varepsilon_{s i X j} & \text { for } & \varepsilon_{s i X j}>0 \\
0 & \text { for } & \varepsilon_{s i X j} \leqslant 0
\end{array}\right.
$$

From equations (49) and (48), the total stay energy for Mode $i$ Type $X$ in stay $j$ is described as follows:

$$
U_{s i X j}=\left\{\begin{array}{lll}
\frac{1}{2} E_{s} A_{s} L_{s}\left(\varepsilon_{s i X j}^{2}-\varepsilon_{s X 0}^{2}\right) & \text { for } \quad \varepsilon_{s i X j} \geqslant 0 \\
0 & \text { for } & \varepsilon_{s i X j} \leqslant 0 .
\end{array}\right.
$$

Note that $\varepsilon_{c X 0}$ and $\varepsilon_{s X 0}$ affect neither the critical load nor the post-buckling path, because they are independent of $q_{m}$ and vanish on differentiation.

\subsubsection{Work done by the load}

The work done by the load $P \mathcal{E}_{i X}$ is defined as the external axial load $P$ multiplied by the corresponding end-shortening $\Delta_{i X} L$ :

$$
P \mathcal{E}_{i X}=P \Delta_{i X} L-P \mathcal{E}_{0 X}
$$

where $P \mathcal{E}_{0 X}$ is the work done by the load before the commencement of each buckling type. Note that, again, this value affects neither the critical load nor the post-buckling path for the same reason as stated in the previous section. 


\subsubsection{Total potential energy function}

The total potential energy is a summation of $U_{c b i}, U_{a b i X}, U_{c a i X}, U_{s i X}$ minus $P \mathcal{E}_{i X}$ :

$$
V_{i X}=U_{c b i}+U_{a b i X}+U_{c a i X}+U_{s i X}-P \mathcal{E}_{i X} .
$$

In the Mode 2 Type $\mathrm{C}$ analysis, higher terms of $\mathrm{P}$ are then truncated as they are not dominant terms in the function and leave the governing equation untractable. For equilibrium, the total potential energy $V_{i X}$ must be stationary with respect to the generalized coordinates $q_{m}$. Therefore, the equilibrium paths can be computed from the condition:

$$
\frac{\partial V_{i X}}{\partial q_{m}}=0
$$

\section{Critical Buckling}

Having formulated the total potential energy, the critical buckling load of the stayed column is investigated using linear eigenvalue analysis. From the earlier work of Hafez et al. [8], it is known that the critical load is divided into three zones in relation to the magnitude of the initial pretension in the stays.

Zone 1 The tension in the stays disappears completely before the external load reaches the buckling load. Therefore, the critical load is exactly the Euler load (Type A buckling).

Zone 2 The strain in the stays becomes zero when the applied load reaches the critical load, i.e. the structure resists buckling until the tension in the stays becomes zero. Thus, all the stays remain effective until buckling, which sends the critical load potentially to a level that is significantly higher than the Euler load (Type C buckling).

Zone 3 The tension in the stays is nonzero at the instant of buckling. As a large amount of the pretension has been introduced, all the stays remain effective for some while after buckling. The value of the critical load falls somewhat as the initial prestress increases because the initial compressive stress in the column diminishes its axial load capacity (Type B buckling).

As the formulation of the model ensures that the profile of the structure maintains perfect symmetry during the fundamental state, a bifurcation point can be observed when $q_{m}=0$. For Type B buckling conventional linear eigenvalue of analysis, i.e. finding when the Hessian matrix for $V_{i X}$ becomes singular, yields the critical load for Zone $3 P_{\text {Zone3 }}^{\mathrm{C} i}$ directly. The details on the process and equations obtained can be seen in Appendix A.

For Zones 1 and 2, it is necessary to consider geometrically nonlinear effects in order to find the critical load, because in these zones, the end-shortening of the column releases the axial energy in the stays during the fundamental stage, which does not allow linear eigenvalue analysis to yield the critical load. Moreover, linear buckling analysis in the 
FEM does not detect the critical load for Zones 1 and 2 either, the analytical method being therefore essential to find the critical load in this range of $T$.

In Zone 1, where the axial energy in the stays is already lost before buckling, this problem can be simply resolved by adopting the Type A buckling energy formulation and then following the same process as for Zone 3. Because in Zone 1 (Type A buckling) all of the stays are slack at the instant of buckling, no substantial changes in the way of determining the critical load are necessary. This analysis yields the critical loads for Zone 1 for Mode $i$ being:

$$
P_{\mathrm{Zone} 1}^{\mathrm{C} i}=\frac{i^{2} \pi^{2} E I}{L^{2}}
$$

As can be seen from this equation, the critical load for Zone 1 is the exactly same as the Euler buckling load $P_{\mathrm{E}}$.

For Zone 2, the critical load can be found from utilizing the condition that the strain in the stays becomes zero at the instant of buckling. As all of the stays are active during the pre-buckling stage, substituting $q_{m}=0$ and $h_{B}=0$ into equation (30) with the adoption of subscript $B$ and solving the equation for $P$ gives the following critical load for Zone 2 for Mode $i$ :

$$
P_{\mathrm{Zone} 2}^{\mathrm{C}}=\frac{T}{b_{p B} E_{s} A_{s} \cos ^{2} \alpha}
$$

where

$$
b_{p B}=\left[2 E_{s} A_{s} \cos ^{3} \alpha+E A\right]^{-1} .
$$

Note that Modes 1 and 2 have the same expression for the Zone 2 critical load. In fact, the instability behaviour in Zone 2 is not a classic bifurcation response: at the point of "buckling" there is a sudden release of the axial energy of the column, forcing the column to buckle, which is immediately followed by the reactivation of the convex side stays as the column displaces laterally.

By plotting the critical loads against $T$, the relationship between the buckling load and the initial prestress, which was discovered by Hafez et al. [8], can be reproduced. This relationship is shown in Figure 11, where $T_{\min }$ represents the initial prestress at the boundary between Zones 1 and 2-the minimum effective pretension required to raise the buckling load above the Euler load - and $P_{\max }$ represents the theoretical maximum buckling load that is observed at the boundary between Zones 2 and 3 .

\subsection{Numerical results}

In this section the aim is to compare theoretical $P_{\max }$ values obtained from the previous section with those from the Hafez model as a benchmark for validation. In the Hafez model, $P_{\max }$ was obtained by the FEM, so that the accuracy of the current model in terms of the critical load can be evaluated. In the Hafez model, $P_{\max }$ was sought with a variation in three parameters: crossarm length, stay diameter and stay Young's modulus; for the current model, the same parameters are varied. The dimensions of the structure used in the Hafez model were as follows: 


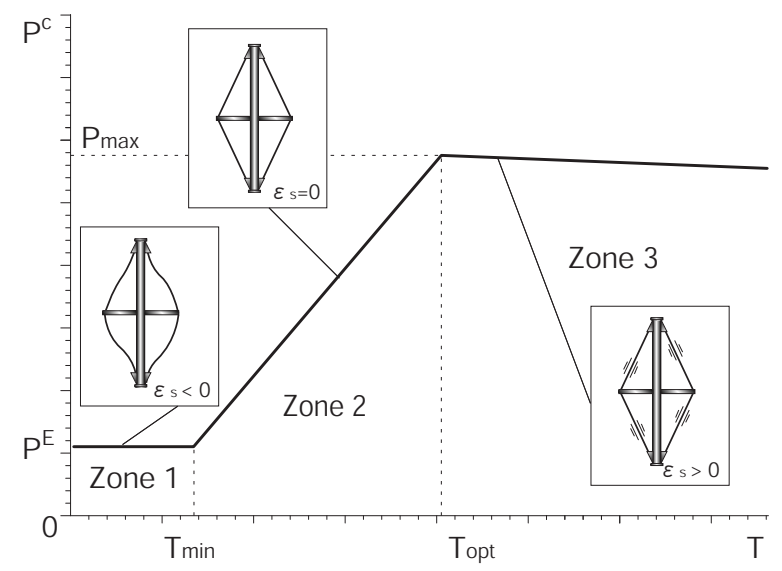

Figure 11: Critical buckling load $P^{\mathrm{C}}$ versus initial prestress $T$.

column Young's modulus:

crossarm Young's modulus:

stay Young's modulus:

column length:

crossarm length:

outside diameter of the column:

inside diameter of the column:

outside diameter of the crossarm:

inside diameter of the crossarm:

stay diameter:

$$
\begin{aligned}
& E=201 \mathrm{kN} / \mathrm{mm}^{2} \\
& E_{a}=201 \mathrm{kN} / \mathrm{mm}^{2} \\
& E_{s}=202 \mathrm{kN} / \mathrm{mm}^{2} \\
& L=3.05 \mathrm{~m} \\
& a=0.305 \mathrm{~m} \\
& \phi_{c o}=38.1 \mathrm{~mm} \\
& \phi_{c i}=25.4 \mathrm{~mm} \\
& \phi_{a o}=38.1 \mathrm{~mm} \\
& \phi_{a i}=25.4 \mathrm{~mm} \\
& \phi_{s}=3.2 \mathrm{~mm} \text { or } \phi_{s}=4.8 \mathrm{~mm} .
\end{aligned}
$$

While the crossarm length $a$ is varied from $0.305 \mathrm{~m}$ to $3.05 \mathrm{~m}$, the stay diameter is fixed to $\phi_{s}=3.2 \mathrm{~mm}$; when the stay Young's modulus $E_{s}$ is varied from $64.8 \mathrm{kN} / \mathrm{mm}^{2}$ to $204 \mathrm{kN} / \mathrm{mm}^{2}$, the stay diameter is fixed to $\phi_{s}=4.8 \mathrm{~mm}$. Figures $12(\mathrm{a})$, (b) and (c) respectively show $P_{\max }$ varying with each parameter along with that of the Hafez model. In the case of the single degree-of-freedom (SDOF) model for Mode 1, there is a certain degree of error shown in Figure 12 between the Hafez and the current model. However, with the two degree-of-freedom (2DOF) model, this error between the two becomes almost negligible. In Mode 2, however, gaps between the Hafez model and the current model can be seen to be more significant. With the three degree-of-freedom (3DOF) model, which is the most sophisticated model presented and therefore is expected to have the least error, some differences are still evident. Although these figures show relatively less good agreement compared with those of Mode 1 , the trend is that increasing the number of freedoms increases the accuracy but with computational expense and analytical complexity.

Considering that the difference between the 2DOF and the 3DOF models is not significant, and that the solutions from the 2DOF model are relatively close to the benchmark solutions, the 2DOF model will be used in order to obtain reasonably accurate solutions for the post-buckling behaviour without it being excessively demanding computationally. 


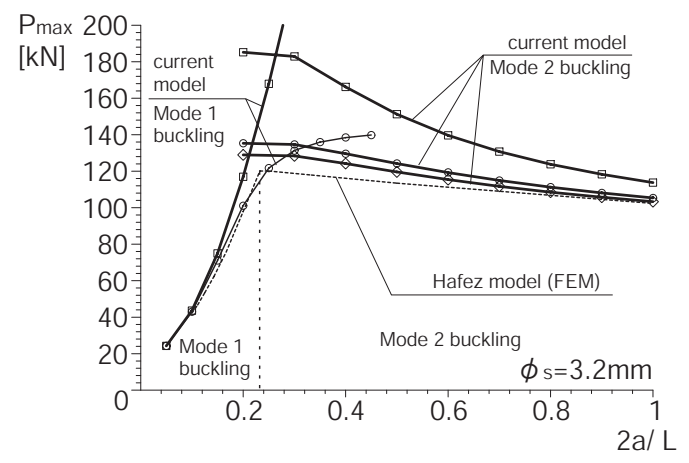

(a) Crossarm length

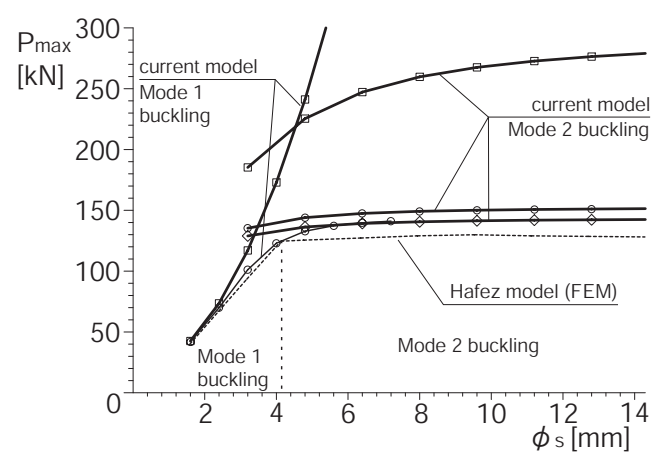

(b) Stay diameter

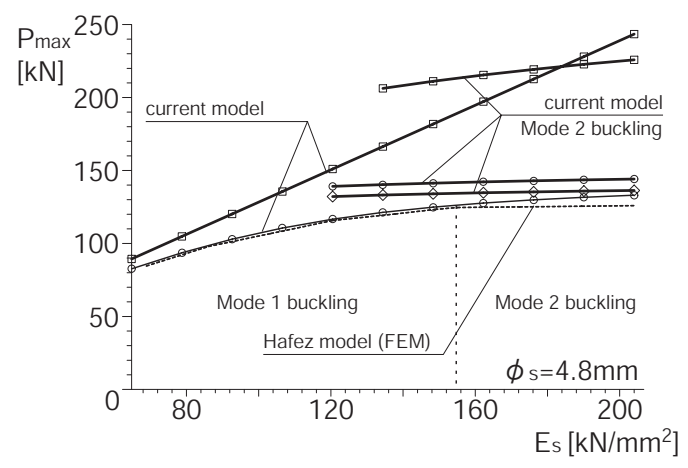

(c) Stay Young's modulus

Figure 12: Comparison of $P_{\max }$ values with those of the Hafez model: (a) varying crossarm length, (b) varying stay diameter, (c) varying Young's modulus. Symbols ( $\square),(\circ)$ and $(\diamond)$ represent the cases of $n=1, n=2$ and $n=3$ respectively.

\section{Post-Buckling Response}

Equation (53) expresses the equilibrium states after buckling, which can be solved using MAPLE. In Mode 1, the same dimensions and properties as in $\S 3.1$ were also applied for the post-buckling analysis, with the stay diameter, $\phi_{s}=4.8 \mathrm{~mm}$ being chosen. The critical buckling loads obtained with those dimensions against the initial prestress are shown in Figure 13. As illustrated, eight points are picked up from each diagram to investigate changes in the post-buckling response as $T$ changes, the selection criteria being expressed in Table 1. 


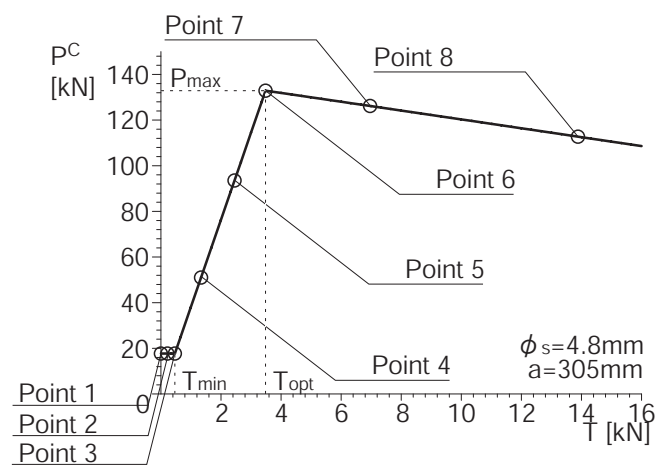

(a) Mode 1

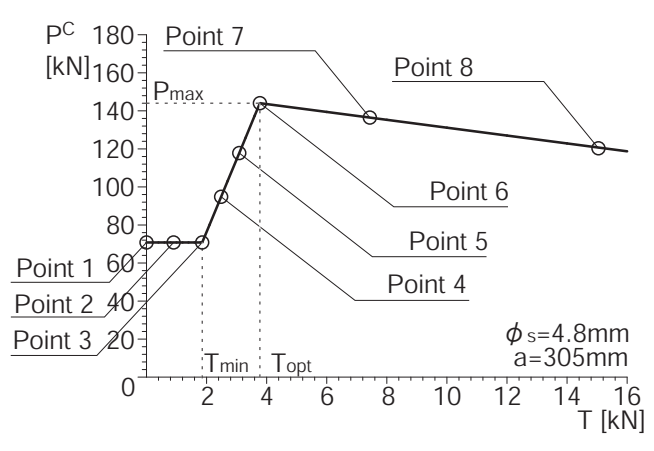

(b) Mode 2

Figure 13: Critical buckling load $P^{\mathrm{C}}$ versus the initial prestress $T$ showing the selected points for the post-buckling investigation.

\begin{tabular}{|c|c|c|c|}
\hline \multirow{2}{*}{ Point } & \multicolumn{3}{|c|}{ Initial prestress $T$} \\
\cline { 2 - 4 } & Criterion expression & Mode 1 $(\mathrm{kN})$ & Mode 2 $(\mathrm{kN})$ \\
\hline 1 & 0 & 0.00 & 0.00 \\
\hline 2 & $T_{\min } / 2$ & 0.23 & 0.93 \\
\hline 3 & $T_{\min }$ & 0.46 & 1.86 \\
\hline 4 & $\left(T_{\mathrm{opt}}-T_{\min }\right) / 3+T_{\min }$ & 1.47 & 2.50 \\
\hline 5 & $2\left(T_{\mathrm{opt}}-T_{\min }\right) / 3+T_{\min }$ & 2.48 & 3.14 \\
\hline 6 & $T_{\mathrm{opt}}$ & 3.48 & 3.78 \\
\hline 7 & $2 T_{\mathrm{opt}}$ & 6.97 & 7.55 \\
\hline 8 & $4 T_{\mathrm{opt}}$ & 13.93 & 15.10 \\
\hline
\end{tabular}

Table 1: Selected points for the post-buckling investigation.

\subsection{Zones of behaviour}

The post-buckling responses for Modes 1 and 2 in each zone are represented in Figures 14 and 15. For Mode 1 the relationship between $P$ and $q_{1}-q_{3}$ is shown. The latter quantity being the normalized horizontal displacement at the column midspan, obtained by evaluating $W_{1}(L / 2) / L$. For Mode 2 the relationship between $P$ and $q_{2}-2 q_{4}$ is shown. The latter quantity being the normalized rotation at the column midspan, obtained by evaluating $\Theta_{2}(L / 2) / 2 \pi$.

For both modes the post-buckling path in Zone 1 has two distinct stages, as shown in (a) and (b) in Figures 14 and 15 respectively; $P$ remains practically at the critical load in Type A buckling (all stays slack) for a while, then the equilibrium path stabilizes with Type C buckling (convex side stays reactivated). Note that, as shown in (b) in Figures 14 


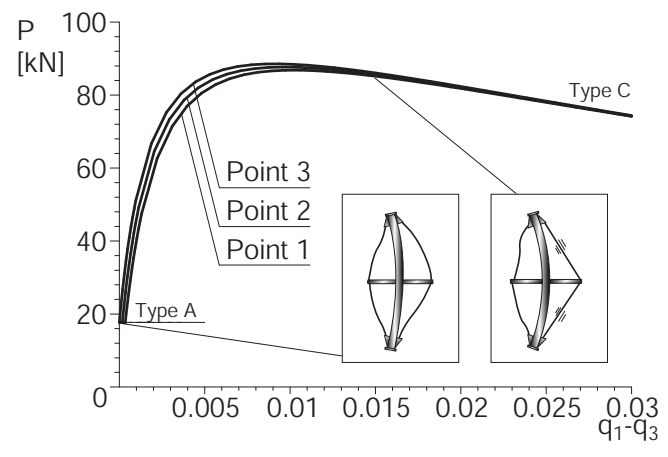

(a) Zone 1

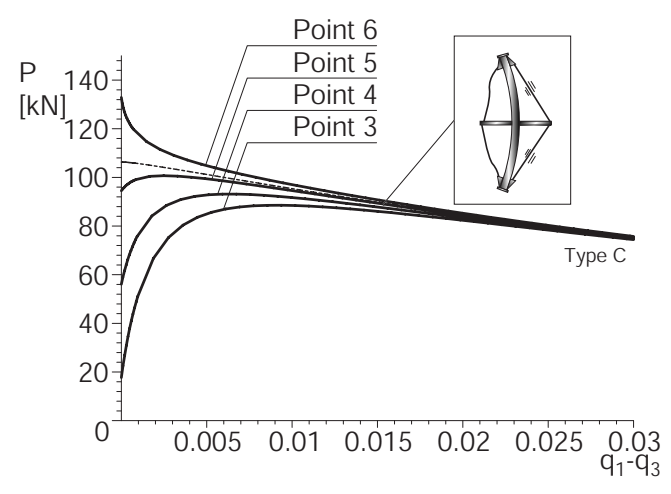

(c) Zone 2

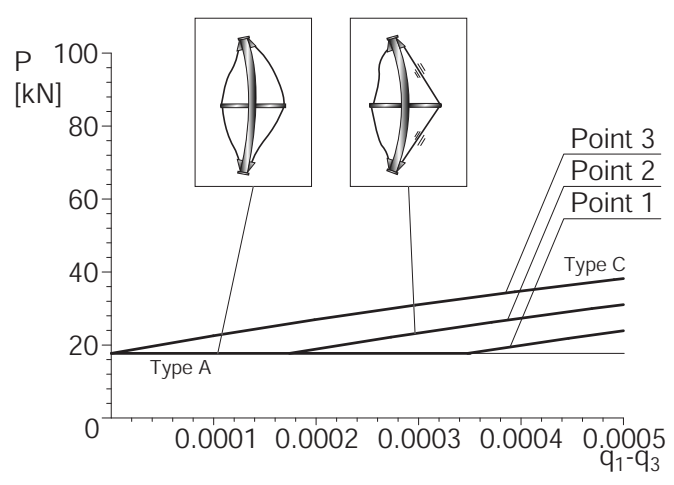

(b) initial part of zone 1

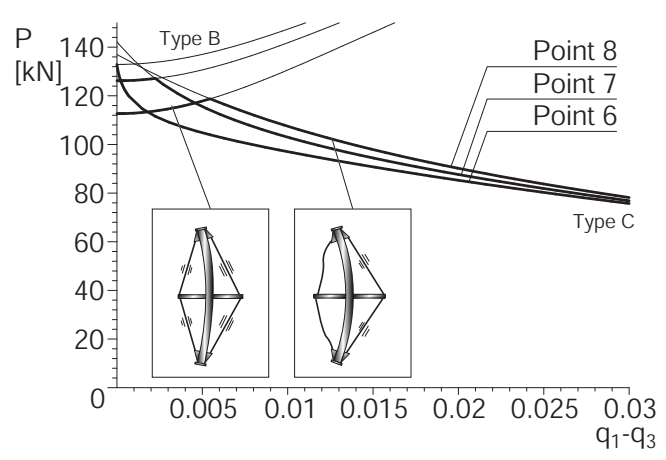

(d) Zone 3

Figure 14: Post-buckling responses for Mode 1 represented by axial load $P$ versus mid-span buckling displacement $q_{1}-q_{3}$.

and 15, the initial flat range becomes shorter as the prestress $T$ is increased.

As shown in the graphs in (c) of Figures 14 and 15, in Zone 2, stable paths can be observed in the initial post-buckling range with relatively low values of the prestress, such as for Points 3, 4, 5, whereas unstable paths can be observed with relatively high values of the prestress, such as for Point 6. The initial prestress at the transition from stability to instability can be found when $T=2.79 \mathrm{kN}$ for Mode 1 . The reason for this transition in Zone 2 can be considered as follows: with a relatively large value of the prestress in Zone 2 , a large amount of the axial energy can be stored in the fundamental state due to the presence of effective axial forces in the stays, which prevents the release of axial energy from the column. Therefore, this excessive amount of the energy is suddenly released at the instant of buckling, which is conjectured to cause the unstable responses. By contrast, with a relatively small value of the prestress, although the axial energy has been able to be stored in the fundamental state, more than in the case of Zone 1, this additional energy can completely be absorbed into the stays after buckling; therefore, stable paths 


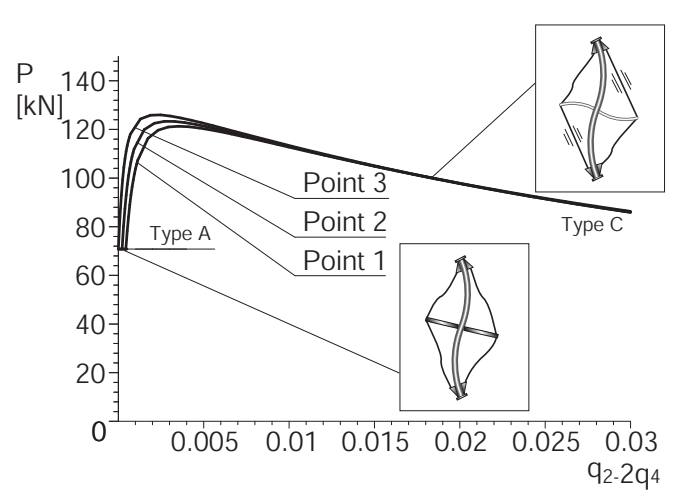

(a) Zone 1

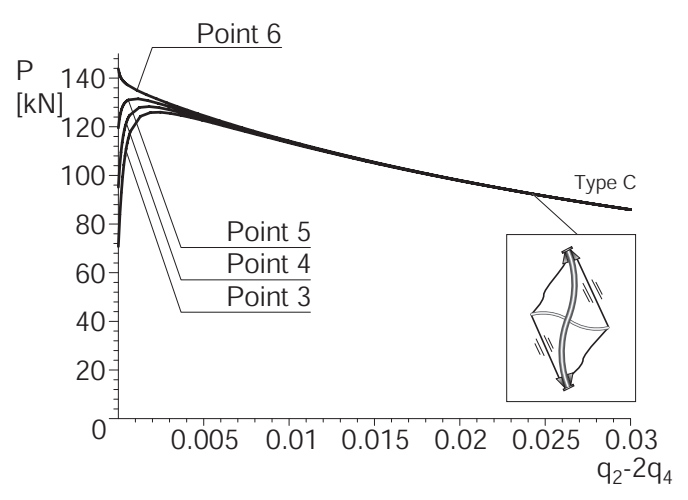

(c) Zone 2

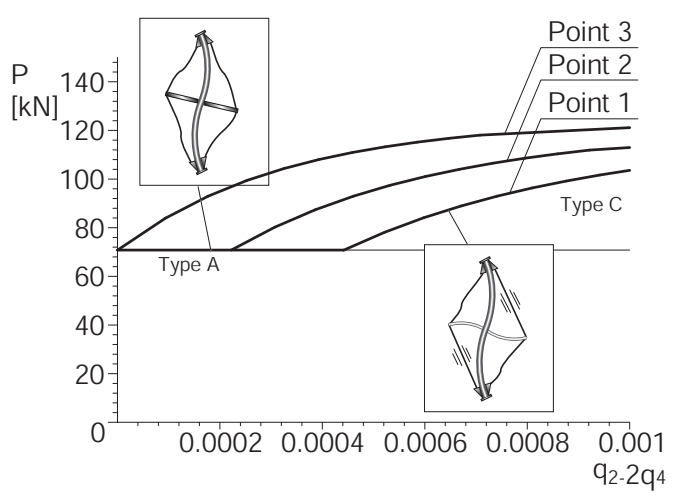

(b) initial part of zone 1

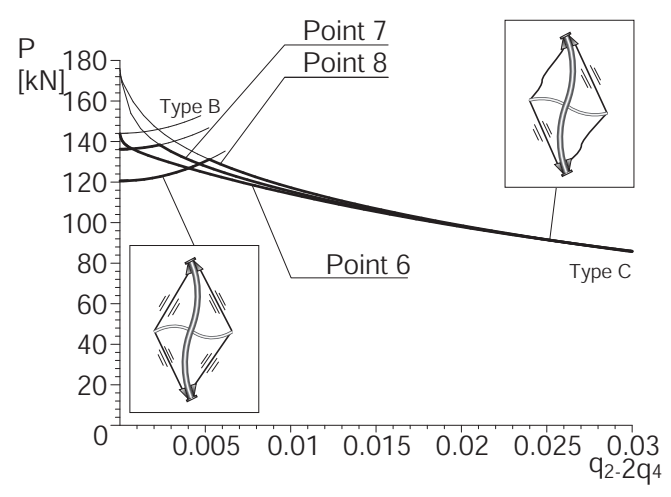

(d) Zone 3

Figure 15: Post-buckling response for Mode 2 represented by axial load $P$ versus midspan buckling rotation $q_{2}-2 q_{4}$.

are seen. Despite this difference within the zone, for any case in Zone 2 post-buckling, the convex side of the stays are active throughout the post-buckling range, with the stays on the concave side being slack, which implies that in Zone 2 the post-buckling response has Type C characteristics.

As shown in the graphs in (d) of Figures 14 and 15, there is also a discontinuity in the post-buckling response in Zone 3 . The load $P$ remains nearly at the critical load in Type B buckling for a while, and this initial stage is followed by Type $\mathrm{C}$ buckling with a sudden loss of the stability; unstable paths are then observed when the concave side stays go slack. The discontinuity of Zone 3 is basically a mirror image of the response in Zone 1, where slackening of stays occurs rather than their reactivation.

For all zones, the only difference between Modes 1 and 2 is the activating stays in the Type $\mathrm{C}$ buckling response: the activating stays are 1 and 2 for Mode 1 , and 1 and 3 for Mode 2. 


\subsection{Validation}

Using the FEM program ABAQUS, a purely numerical model was developed and the postbuckling response was revealed by a nonlinear Riks analysis to validate the results presented in the previous section. In this procedure, the column and the crossarm were modelled as beam elements and the stays were modelled as truss elements. The "No compression option", which prevents any compression force entering the truss elements, was also adopted to simulate any slackening in the stays. Furthermore, it is essential in this type of nonlinear analysis to introduce an imperfection. In the current study, this was achieved through using the Euler buckling displacement generated by eigenvalue analysis. The magnitude of the imperfection was intended to be deliberately small such that the perfect response would be approximated. To trigger Mode 1 , an out-of straightness of $L / 10000$ was imposed at the middle of the column. To trigger Mode 2, an out-of straightness of $L / 14142$ was imposed at the quarter and the three-quarter points along the column such that the horizontal displacement at those points would be the same as that in Mode 1 .

\subsubsection{Comparisons}

Figures 16 and 17 show the post-buckling responses from the FEM along with those from the analytical models at Points 1, 3, 6, 7 and 8. As can be seen in Figure 16, for Mode 1, the post-buckling paths of the FEM model almost coincide with those of the analytical model. However, Figure 17 shows less good agreement between the FEM and the analytical models in Mode 2. Regardless of the less good agreement in Mode 2, the same trend can still be detected from these two models; therefore, the analytical models for Mode 2 are still useful for predicting the qualitative buckling behaviour.

From this comparison, it can be said that the Mode 1 buckling of the stayed column can be modelled as the current 2DOF analytical model with great accuracy. Also, it can be said that with the current analytical 2DOF model for Mode 2, the approximated post-buckling response can be obtained; however it has to be admitted that the analytical model involves a certain discrepancy with the numerical model. This inaccuracy can be reduced by increasing the number of degrees of freedom, but this process is computationally demanding as discussed earlier.

\section{Design Implications and Further Work}

Some implications for design can be deduced from the responses that have been presented. Firstly, when the prestress is relatively low, such as in Zone 1 and in an initial part of Zone 2 in which stable post-buckling paths can be seen, the design load can be set on the basis of the maximum strength, which can be larger than the critical load as long as plasticity and excessive deflection can be avoided. However, when the prestress is relatively high, such as in Zone 3 and the rest of Zone 2 in which unstable paths can be seen, the design load should be much less than the critical load because with imperfections, the maximum strength of the structure usually becomes significantly lower than the critical load and elastic failure 


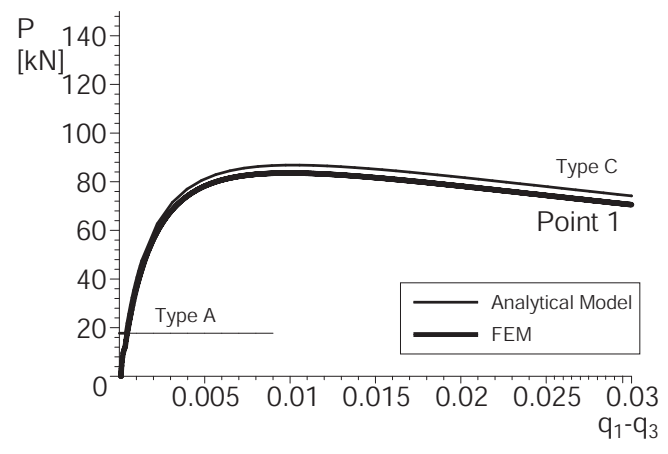

(a) Zone 1

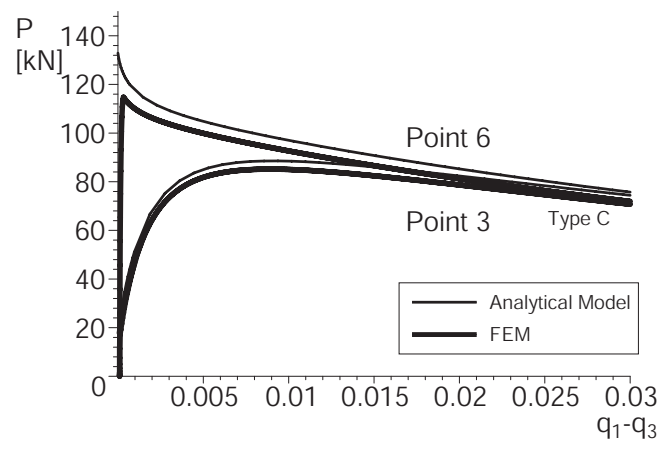

(b) Zone 2

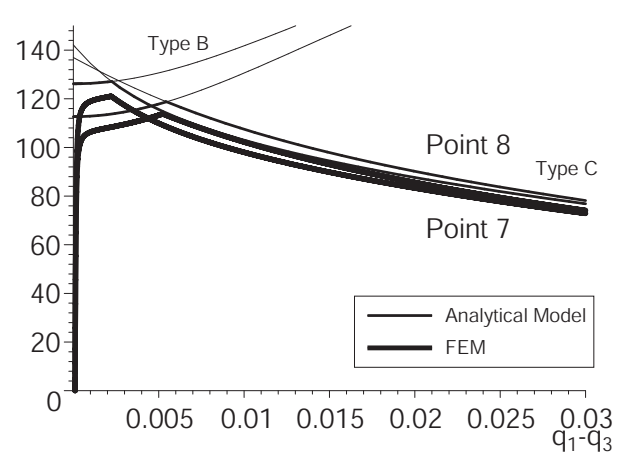

(c) Zone 3

Figure 16: Equilibrium paths for Mode 1 comparing the FEM and the analytical models.

ensues. The margin between the maximum strength and the design load should be decided by imperfection sensitivity studies, which the authors are currently undertaking.

Also, with a very large amount of prestressing, much higher than $T_{\text {opt }}$, the critical load may become relatively reliable for the collapse load because large amounts of buckling displacement are necessary before unstable post-buckling occurs. Hence, it can be said that, when greater stability is required for a structure, introducing a large value of the prestress is strongly recommended. However, it should be noted that a large amount of prestressing would require a significant increase in the cross sectional area of the structural components to counteract potential plasticity effects, which may lead this component to being less cost-effective.

The current studies do not account for geometric imperfections nor plasticity of steel. Hence, it does not reflect the actual response of the stayed column, although the model has revealed the principle of the post-buckling response in relation to the initial response in the ideal situation. The importance of geometric imperfections was already discussed in earlier works $[9,10,11,12]$. To predict the more realistic response of the structure, it is 


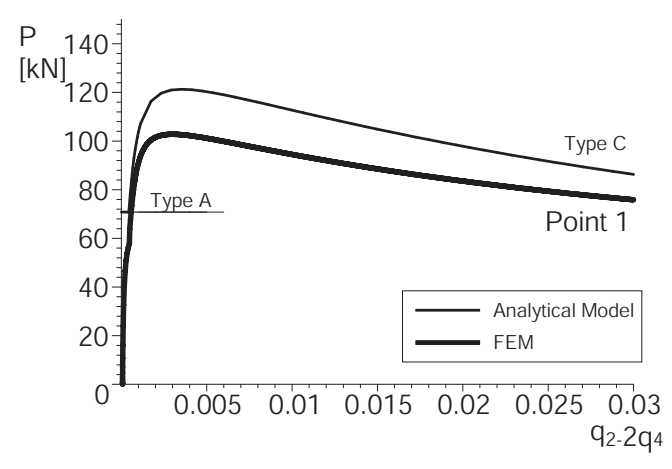

(a) Zone 1

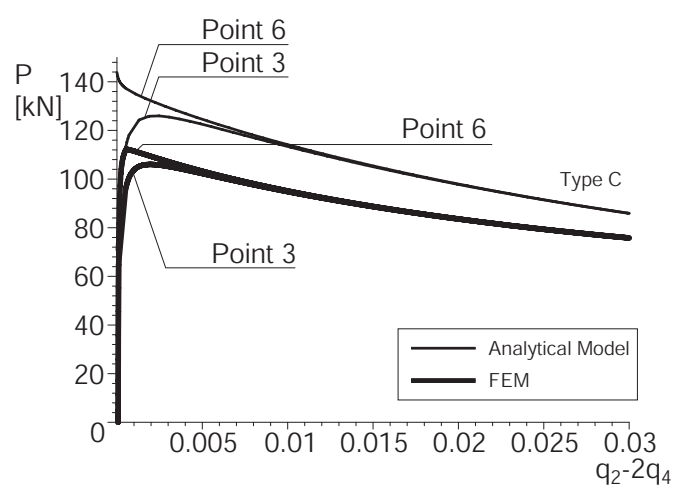

(b) Zone 2

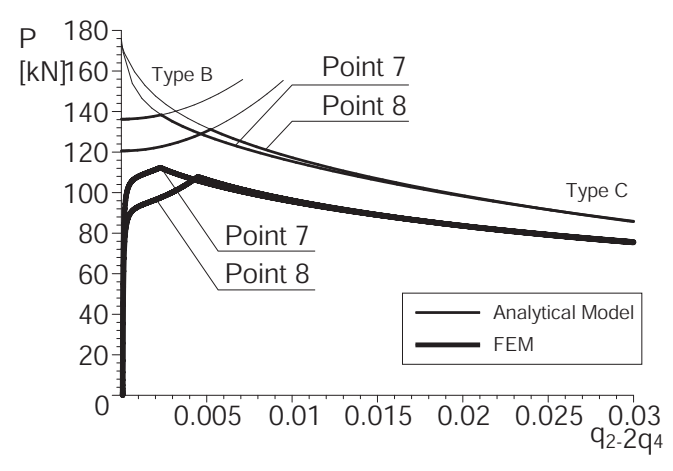

(c) Zone 3

Figure 17: Equilibrium paths for Mode 2 comparing the FEM and the analytical models.

necessary to incorporate imperfections and the plastic behaviour of each component into the current model, and the authors are currently working on this.

Another aspect that is currently neglected is the effect of stress relaxation that may occur due to creep and changes in the ambient temperature with the stays and the column changing their lengths, and thereby their internal forces, causing a change in their stress state. This may manifest itself in stay relaxation; in Zone 3, where the optimal prestress is now considered to be located, relaxation may change the response of the column by reducing the prestress from where the column has a relatively stable initial post-buckling (points 7 and 8) to purely unstable post-buckling (towards point 6). This adverse effect from stress changes would be a key sensitivity to focus on in future work. If this sensitivity is significant, it would also be suggested that designers should take into account both the initial prestress and the effective value of prestress after a long time period. Of course, the situation would become more complicated if materials of different coefficients of thermal expansion are used in the column and the stays respectively, or if the temperature changes are non-uniform within the whole component. 
Interactive buckling is another issue to be tackled. This is a phenomenon in which different types of buckling modes occur simultaneously. Figure 12 shows that there is a boundary between Modes 1 and 2; it is one of the places where interactive buckling possibly occurs [13]. From work on other structural components [16], it has been known that interactive buckling is also triggered by the interaction between local and global instabilities. Catastrophic failure can often be observed with this type of instability, and therefore needs further investigation.

Experimental studies focusing on the post-buckling response are also necessary in order to validate the analytical and the numerical models. This process becomes vital, especially when the structure is more complicated than the current structure and therefore it becomes more difficult to formulate analytical models. Moreover, the current modelling is limited to two-dimensional (2D) behaviour, it may become important to develop three-dimensional (3D) models. Recent work $[17,18]$ has used 3D modelling to address 3D collapse responses with a variety of structural configurations and boundary conditions, and with different levels of the prestress, but certain other stability issues need to be investigated in 3D such as local buckling mentioned above and torsional buckling if, for example, open sections are used instead of closed sections for the main column component.

The work outlined above could be used as a basis to produce design guidance. Currently, codes of practice, such as the European design code for steel structures [19], are lacking in the design procedures for such potentially efficient and cost-effective structures; consequently, case and sensitivity studies in conjunction with engineering judgement are necessary to design the stayed column in practice. Establishing such guidance for the stayed column will facilitate designers to adopt this structural component more effectively.

\section{Concluding Remarks}

The post-buckling behaviour of the prestressed steel stayed column has been investigated using the Rayleigh-Ritz method. It has been shown that the post-buckling response is strongly linked to the zone distinction of the critical loads that was found by Hafez et al. [20] for the first two buckling modes. In Zone 1, the response is initially similar to that of Euler buckling, which is followed by a rather stable path thanks to the reactivation of the stays. In Zone 2, the critical load is increased to more than the Euler load and either a stable or an unstable path emerges after buckling, depending on values of the prestress and other structural properties. In Zone 3, the critical load reaches its theoretical maximum, and the post-buckling path becomes unstable, after an initially flat but slightly stable response, due to some of the stays slackening. These results have been validated using the FEM. It has been shown that the current analytical model for Mode 1 has excellent agreement with the FEM model; however it is less accurate for Mode 2 when compared to Mode 1, even though the model is still useful to find approximate post-buckling responses for that mode. Design implications have also been deduced from those results; it has been stated that any design loads should be carefully determined with consideration for the post-buckling response in order to achieve safe and efficient designs because the results 
have shown that the maximum strength depends primarily on the prestress rather than the classically evaluated buckling load.

\section{A Appendix: Hessian matrix for Zone 3}

When $m=2$ and in Mode 1, the critical load for Zone 3 can be obtained through calculating the following determinant of the Hessian Matrix:

$$
\left|\begin{array}{cc}
\frac{\partial^{2} V_{1 B}}{\partial q_{1}^{2}} & \frac{\partial^{2} V_{1 B}}{\partial q_{1} \partial q_{3}} \\
\frac{\partial^{2} V_{1 B}}{\partial q_{3} \partial q_{1}} & \frac{\partial^{2} V_{1 B}}{\partial q_{3}^{2}}
\end{array}\right|=0 .
$$

Each component of the matrix can be expressed as

$$
\begin{aligned}
\frac{\partial^{2} V_{1 B}}{\partial q_{1}^{2}} & =\chi_{p 11} P+\chi_{t 11} T+\chi_{11}, \\
\frac{\partial^{2} V_{1 B}}{\partial q_{1} \partial q_{3}}=\frac{\partial^{2} V_{1 B}}{\partial q_{3} \partial q_{1}} & =\chi_{p 13} P+\chi_{t 13} T+\chi_{13}, \\
\frac{\partial^{2} V_{1 B}}{\partial q_{3}^{2}} & =\chi_{p 33} P+\chi_{t 33} T+\chi_{33} .
\end{aligned}
$$

The coefficients of the above equations are as follows:

$$
\begin{aligned}
\chi_{p 11} & =\frac{a\left[8 b_{p B} E_{s} A_{s}\left(b_{11 B}-2 \cos ^{2} \alpha\right) \cos ^{3} \alpha+b_{p B} E A\left(4 b_{11 B}-\pi^{2}\right)-4 b_{11 B}\right]}{\tan \alpha}, \\
\chi_{t 11} & =\frac{2 a\left(8 \cos ^{2} \alpha-\pi^{2}\right) \cos \alpha}{\tan \alpha}, \\
\chi_{11} & =\frac{\left(64 a^{2} E_{s} A_{s} \cos ^{3} \alpha+E I \pi^{4}\right) \tan \alpha}{4 a} \\
\chi_{p 13} & =\frac{2 a\left[2 b_{p B} E_{s} A_{s}\left(4 \cos ^{2} \alpha+b_{13 B}\right) \cos ^{3} \alpha+b_{13 B}\left(b_{p B} E A-1\right)\right]}{\tan \alpha}, \\
\chi_{t 13}= & \frac{-16 a \cos ^{3} \alpha}{\tan \alpha}, \\
\chi_{13}= & -16 a E_{s} A_{s} \sin \alpha \cos ^{2} \alpha, \\
\chi_{p 33}= & \frac{a\left[8 b_{p B} E_{s} A_{s}\left(b_{33 B}-2 \cos ^{2} \alpha\right) \cos ^{3} \alpha+b_{p B} E A\left(4 b_{33 B}-9 \pi^{2}\right)-4 b_{33 B}\right]}{\tan \alpha}, \\
\chi_{t 33}= & \frac{2 a\left(8 \cos ^{2} \alpha-9 \pi^{2}\right) \cos \alpha}{\tan \alpha}, \\
\chi_{33}= & \frac{\left(64 a^{2} E_{s} A_{s} \cos ^{3} \alpha+81 E I \pi^{4}\right) \tan \alpha}{4 a},
\end{aligned}
$$


where

$$
\begin{aligned}
& b_{11 B}=\left[4 E_{s} A_{s}\left(3 \cos ^{2} \alpha-2\right) \cos ^{3} \alpha-\pi^{2}\left(\frac{\pi^{2} E I}{L^{2}}-\frac{E A}{4}\right)\right]\left[2 E_{s} A_{s} \cos ^{3} \alpha+E A\right]^{-1}, \\
& b_{33 B}=\left[4 E_{s} A_{s}\left(3 \cos ^{2} \alpha-2\right) \cos ^{3} \alpha-9 \pi^{2}\left(\frac{9 \pi^{2} E I}{L^{2}}-\frac{E A}{4}\right)\right]\left[2 E_{s} A_{s} \cos ^{3} \alpha+E A\right]^{-1}, \\
& b_{13 B}=-2\left[4 E_{s} A_{s}\left(3 \cos ^{2} \alpha-2\right) \cos ^{3} \alpha+\frac{15 \pi^{4} E I}{L^{2}}\right]\left[2 E_{s} A_{s} \cos ^{3} \alpha+E A\right]^{-1} .
\end{aligned}
$$

The critical load for Mode 2 can also be obtained by following the same process as that of Mode 1, even though the expressions for Mode 2 are more complicated, reflecting the extra complexity in the model for that buckling mode.

\section{References}

[1] S. A. L. de Andrade, P. C. G. da S. Vellasco, and J. G. S. da Silva. Concepcão e projecto estrutural do palco principal do Rock in Rio III. Construçao Magazine, 6:4-11, 2003. (in Portuguese).

[2] S. A. L. de Andrade, P. C. G. da S. Vellasco, and J. G. S. da Silva. Sistema construtivo e montagem estrutural do palco principal do Rock in Rio III. Construçao Magazine, 7:30-35, 2003. (in Portuguese).

[3] K-H. Chu and S. S. Berge. Analysis and design of struts with tension ties. J. Struct. Div. ASCE., 89(ST 1):127-163, 1963.

[4] H. R. Mauch and L. P. Felton. Optimum design supported by tension tension ties. J. Struct. Div. ASCE., 93(ST 3):201-220, 1967.

[5] R. J. Smith, G. T. McCaffrey, and J. S. Ellis. Buckling of a single-crossarm stayed column. J. Struct. Div. ASCE., 101(ST 1):249-268, 1975.

[6] M. C. Temple. Buckling of stayed columns. J. Struct. Div. ASCE., 103(ST 4):839-851, 1977.

[7] E. Belenya. Prestressed Load-Bearing Metal Structures. Mir Publishers, Moscow, 1977.

[8] H. H. Hafez, M. C. Temple, and J. S. Ellis. Pretensioning of single-crossarm stayed columns. J. Struct. Div. ASCE., 105(ST 2):359-375, 1979.

[9] K. C. Wong and M. C. Temple. Stayed column with initial imperfection. J. Struct. Div. ASCE., 108(ST 7):1623-1640, 1982. 
[10] S. L. Chan, G. Shu, and Z. Lü. Stability analysis and parametric study of pre-stressed stayed columns. Eng. Struct., 24:115-124, 2002.

[11] M. C. Temple, M. V. Prakash, and J. S. Ellis. Failure criteria for stayed columns. J. Struct. Div. ASCE., 110(11):2677-2689, 1984.

[12] E. A. Smith. Behavior of columns with pretensioned stays. J. Struct. Div. ASCE., 111(5):961-972, 1985.

[13] J. M. T. Thompson and G. W. Hunt. A general theory of elastic stability. Wiley, London, 1973.

[14] A. Heck. Introduction to Maple. Springer, New York, USA, 2003.

[15] ABAQUS. ABAQUS/Standard: User's manual version 6.3. Hibbitt, Karlsson \& Sorensen, Inc., Pawtucket, USA, 2002.

[16] G. W. Hunt and M. A. Wadee. Localization and mode interaction in sandwich structures. Proc. R. Soc. A, 454:1197-1216, 1998.

[17] J. Y. R. Liew and J.-J. Li. Advanced analysis of pre-tensioned bowstring structures. Int. J. Steel Struct., 6(2):153-162, 2006.

[18] R. R. de Araujo, S. A. L. de Andrade, P. C. G. da S. Vellasco, J. G. S. da Silva, and L. R. O. de Lima. Structural response of pre-stressed stayed steel columns. Stability and Ductility of Steel Struct., pages 241-248, 2006.

[19] EN1993-1-1. Eurocode 3: Design of steel structures - Part 1-1: General rules and rules for buildings. CEN, 2005.

[20] I. A. Hathout, M. C. Temple, and J S. Ellis. Buckling of space stayed columns. J. Struct. Div. ASCE., 105(ST 9):1805-1822, 1979. 